\title{
Improvement of Nuclear Facilities DNP3 Protocol Data Transmission Security using Super Encryption BRC4 in SCADA Systems
}

\author{
Eko Hadiyono Riyadi ${ }^{1,2}$, Agfianto Eko Putra ${ }^{1}$, Tri Kuntoro Priyambodo ${ }^{\text {Corresp. } 1}$ \\ 1 Department of Computer Science and Electronics, Universitas Gadjah Mada, YOGYAKARTA, DIY, Indonesia \\ 2 Assessment Center for Nuclear Installation and Material, Nuclear Energy Regulatory Agency (BAPETEN), Jakarta, Indonesia, Jakarta, Indonesia \\ Corresponding Author: Tri Kuntoro Priyambodo \\ Email address: mastri@ugm.ac.id
}

Background. Data transmissions using the DNP3 protocol over the internet in SCADA systems are vulnerable to interruption, interception, fabrication, and modification through man-in-the-middle (MITM) attacks. This research aims to improve the security of DNP3 data transmissions and protect them from MITM attacks.

Methods. This research describes a proposed new method of improving DNP3 security by introducing BRC4 encryption. This combines Beaufort encryption, in which plain text is encrypted by applying a polyalphabetic substitution code based on the Beaufort table by subtracting keys in plain text, and RC4 encryption, a stream cipher with a variable-length key algorithm. This research contributes to improving the security of data transmission and accelerating key generation.

Results. Tests are carried out by key space analysis, correlation coefficient analysis, information entropy analysis, visual analysis, and time complexity analysis. The results show that to secure encryption processes from brute force attacks, a key of at least 16 characters is necessary. IL data correlation values were IL1 $=-0.010$, IL2 $=0.006$, and IL3 $=0.001$, respectively, indicating that the proposed method (BRC4) is better than the Beaufort or RC4 methods in isolation. Meanwhile, the information entropy values from IL data are IL1 $=7.84$, IL2 $=7.98$, and IL3 $=7.99$, respectively, likewise indicating that the proposed method is better than the Beaufort or RC4 methods in isolation. Both results also show that the proposed method is secure from MITM attacks. Visual analysis, using a histogram, shows that ciphertext is more significantly distributed than plaintext, and thus secure from MITM attacks. The time complexity analysis results show that the proposed method algorithm is categorized as linear complexity. 
1 Improvement of Nuclear Facilities DNP3 Protocol Data

2 Transmission Security using Super Encryption BRC4

3 in SCADA Systems

4

5

6

7

\section{8}

\author{
EH. Riyadi ${ }^{12}$, Agfianto E. Putra ${ }^{1}$, Tri K. Priyambodo ${ }^{1}$ \\ ${ }^{1}$ Computer Science and Electronics Department, Universitas Gadjah Mada, Yogyakarta, \\ Indonesia \\ ${ }^{2}$ Assessment Center for Nuclear Installation and Material, Nuclear Energy Regulatory Agency \\ (BAPETEN), Jakarta, Indonesia \\ Corresponding Author: \\ Tri K. Priyambodo ${ }^{1}$ \\ Universitas Gadjah Mada, Yogyakarta, Indonesia \\ Email address: mastri@ugm.ac.id
}

\title{
Abstract
}

Background. Data transmissions using the DNP3 protocol over the internet in SCADA systems are vulnerable to interruption, interception, fabrication, and modification through man-in-themiddle (MITM) attacks. This research aims to improve the security of DNP3 data transmissions and protect them from MITM attacks.

Methods. This research describes a proposed new method of improving DNP3 security by introducing BRC4 encryption. This combines Beaufort encryption, in which plain text is encrypted by applying a poly-alphabetic substitution code based on the Beaufort table by subtracting keys in plain text, and RC4 encryption, a stream cipher with a variable-length key algorithm. This research contributes to improving the security of data transmission and accelerating key generation.

Results. Tests are carried out by key space analysis, correlation coefficient analysis, information entropy analysis, visual analysis, and time complexity analysis. The results show that to secure encryption processes from brute force attacks, a key of at least 16 characters is necessary. IL data correlation values were IL1 $=-0.010$, IL2 $=0.006$, and IL3 $=0.001$, respectively, indicating that the proposed method (BRC4) is better than the Beaufort or RC4 methods in isolation. Meanwhile, the information entropy values from IL data are IL1 $=7.84$, IL2 $=7.98$, and IL3 $=$ 7.99, respectively, likewise indicating that the proposed method is better than the Beaufort or RC4 methods in isolation. Both results also show that the proposed method is secure from MITM attacks. Visual analysis, using a histogram, shows that ciphertext is more significantly distributed than plaintext, and thus secure from MITM attacks. 
39 The time complexity analysis results show that the proposed method algorithm is categorized as 40 linear complexity.

41

42

43

44

45

46

47

48

49

50

51

52

53

54

55

56

57

58

59

60

61

62

63

64

65

66

67

68

69

70

71

72

73

74

75

76

77

78

\section{Introduction}

Supervisory control and data acquisition (SCADA) is a control system architecture comprising computers, networked data communications, and graphical user interfaces (GUI) for high-level process supervisory management, as well as other peripheral devices such as programmable logic controllers (PLC) and discrete proportional-integral-derivative (PID) controllers, which are used to interface with machinery or process plants.

SCADA allows operators to change setpoint data from a distance, monitor processes, and obtain measurement information. It consists of three components: a remote terminal unit (RTU) to collect data from the sensor and remote device, a master terminal unit (MTU) equipped with a Human Machine Interface (HMI) for monitoring and control, and communication infrastructure to connect components [1]-[3]. SCADA requires an industrial network protocol—a real-time communication protocol made to connect interface communication systems and instruments - to communicate with controlled devices.

In SCADA, security is an important factor [4], especially in critical industrial infrastructure.

SCADA security systems connected to the internet are closely related to cybersecurity, and thus require special attention in critical industries. The development of cyberinfrastructure can improve the interconnection and security of smart networks [5]; indeed, several designs have sought to increase their investigative abilities [6].

Cyberattacks have damaged critical facilities, including nuclear facilities [7]. One example of protocol implementation is found in nuclear power plants (NPP), smart grid electricity facilities [8]-[10] that are strictly regulated owing to safety and security considerations. It is thereby necessary to ensure the safety and security of SCADA implementation [11], [12]. Several NPPs have been found vulnerable to cyberattacks, and various attempts have been made to improve nuclear security, using systematic [8] and dynamic mapping systems to determine which assets are most vulnerable to cyberattacks [9].

Famous industrial network protocols include Modbus [11], ICCP/TASE.2 [13], Distributed Network Protocol/DNP3 [12], [14], [15], and OPC [16]. Each has its unique characteristics, as well as its own unique methods for verifying the integrity and data security. The specific requirements of industrial networks often make protocols particularly vulnerable to interference [2], [15], [17]. Among the above-mentioned standard network protocols, DNP3 is the most popular.

Initially, DNP3 was designed for local network communication between MTU and RTU, or between RTU and IED. As most users implement DNP3 to communicate serially, the protocol was developed to work through routable protocols such as TCP/IP [18]. As a message protocol, DNP3 was developed to work over IP, thus making RTU communication more accessible via modem networks [19]. The advantages of DNP3 over other protocols include its reliability, efficiency, and real-time transference of data, as well as its implementation of several standard 
79

80

81

82

83

84

85

86

87

88

89

90

91

92

93

94

95

96

97

98

99

100

101

102

103

104

105

106

107

108

109

110

111

112

113

114

115

116

117

data formats and support for data synchronization (both of which make real-time transmission more efficient and reliable) [1], [20], [21].

However, the connection of SCADA systems to the internet network through the DNP3 protocol is also problematic, as its connections are potentially open to vulnerability loopholes. Such vulnerability can be used by attackers to steal the transmitted data. Furthermore, attackers may interrupt, intercept, fabricate, and modify the data, which would also hamper SCADA [8]-[10], [21]-[29].

It may therefore be concluded that data transmissions through DNP3 protocols in SCADA systems within critical industries are vulnerable to man-in-the-middle (MITM), brute force, eavesdropping, etc. This research aims to improve the security of plain data transmission through DNP3 protocols from the above-mentioned attacks.

\section{Related Works}

Although SCADA is widely used due to its rapid development, recent studies have highlighted its vulnerabilities from a cyber-security and cyber-physical security perspective [14], [15], [22], [23], [27], [28], [30]-[37]. The threat of cyberattacks looms over SCADA systems that communicate with network protocols [22], [23], [27], [34], [38]. As such, A. Hou et al. investigated the detection of attacks within SCADA systems and network protocols, including the possibility of detecting attacks in smart grids using Dirichlet [39]. Similarly, Mantere et al. analyzed the detection of anomalies that could breach security[22].

The analysis and detection of network anomalies must be carried out continuously and periodically, perhaps through modeling and simulation using the OPNET Modeler method [39]. Sniffing and DDOS attacks may be detected in smart grids with Novel IDS technology[26]. A simulation test was conducted using NS-2 in the Novell IEEE802.15.4 protocol, finding that security performance improved between 95.5 and $97 \%$ [3].

Several studies related to the security of DNP3 protocols in SCADA systems have also been conducted. Such studies have investigated how security systems can be implemented, tested, or developed in SCADA systems' DNP3 protocol. One such study tested communication protocol using DNPSec [1]. Shahzad, meanwhile, tested three layers of DNP3 using dynamic cryptographic buffers, showing that it could reduce the effectiveness of attacks and improve security [40]. Research about the application of security authentication using a Tamarin model in a smart grid, meanwhile, showed that a DNP3-SA protocol meets security standards [12]. The testing of the DNP3 protocol was also proposed by developing a Linux-based firewall for industrial control systems, especially in the power sector, using the U32 byte matching feature [36]. Research related to broadcast security messages from MTU to several other stations in DNP3-SAB protocol in SCADA systems showed that broadcasts can be secured from several attack vectors, such as modification, injection, spoofing, and replay [34]. A vulnerability analysis of the DNP3 protocol was carried out by observing specific surface attacks within the Data Link and Application layers [37]. 
118 Elsewhere, DNP3 safety analysis was conducted using a Colored Petri Nets model and Linear

119 Discriminant Analysis, finding that the system can successfully detect and reduce abnormal

120 activity [41]. The development of a security assessment framework for cyber-physical systems

121 was conducted by investigating the DNP3 protocol's resistance to attacks, including passive

122 network monitoring, response replay requirements, rogue interoperations, buffer flooding, and

123 TCP veto [15]. Through vulnerability and penetration testing, MITM attacks were modeled using

124

125

126

127

128

129

130

131

132

133

134

135

136

137

138

139

140

141

142

143

144

145

146

147

148

149

150

151

152

153

154

155 grid technology to evaluate cybersecurity threats to SCADA systems implementing DNP3 protocols [42]. Two attack scenarios were used: an unsolicited message attack and injection data collection [43].

Cryptography aims to ensure that the data sent is correct and accessed by the right people.

Research on secure network protocols was carried out by applying encryption using the DiffieHellman method [44]. Premnath et al., testing the NTRU cryptographic method, found that it has a faster runtime than RSA at the same security level [45]. Testing of new encryption methods was also carried out using an i-key to implement a secure communication system [46]. The use of an i-key as a cryptographic protocol during dynamic re-locking was carried out by testing and simulating the IEEE802.1 $\mathrm{x}$ standard protocol using WEP system encryption [47]. It is claimed that such research might prevent MITM attacks because the decryption processes can only be executed by authorized senders and recipients, i.e. those who update the i-key.

The security of the DNP3 protocol in SCADA was improved using the bump-in-the-wire method, which consists of key distribution, cryptography, and intrusion detection [48]. Using IDS, Jain tested a combination of syntactic and semantic detection techniques, with the DiffieHelman method used for locking and DNPSec used as communication protocol. Testing showed that DNP3 security can be improved through efficient management and crypto-key distribution, while DNPSec can identify other packets on the DNP3 network [1].

Furthermore, research into dynamic cryptographic buffers was conducted using eight remote units divided into two stations (S-bed1) and sixteen isolated groups divided into two stations (Sbed2). The study was successful in preventing an MITM attack [49]. Communication between MTU and RTU was subsequently verified using openDNP3, with Scapy equipment used for packet manipulation and penetration testing. The results showed that OpenDNP3 v1.1.0 supports the prevention of attacks that affect confidentiality, such as MITM [50]. Protecting the integrity and confidentiality of data is important for any network protocol [51].

Elliptic cryptography and hash functions have been developed to analyze performance, as has the 3PAKE protocol, and third-party key exchange authentication. Tests were carried out using AVISPA simulator software, showing that the protocol can efficiently prevent active or passive attacks [52]. Sankhanil Dey et al. analyzed the crypto security of four-bit and eight-bit crypto Sboxes, finding that S-box crypto security is better than DES and AES [53].

\subsection{Comparison to Other Hybrid Cipher Approaches}

Peer] Comput. Sci. reviewing PDF | (CS-2021:03:59198:1:0:NEW 5 Aug 2021) 
156 To prevent cyberattacks, any data communication system must include strong data transmission

157

158

159

160

161

162

163

164

165

166

167

168

169

170

171

172

173

174

175

176

177

178

179

180

181

182

183

184

185

186

187

188

189

190

191

192

193

194

195

security, perhaps using cryptography [54]. Every cryptographic scheme has its own strengths and weaknesses, and thus the application of a single cryptographic technique has severe shortcomings. To secure data, without compromising security, a cost-effective symmetric encryption method is often used. However, in such processes, proper key distribution is problematic [55]. An asymmetric scheme has potential. Unfortunately, however, the process is slower and consumes more computer resources than symmetric encryption. The integration of several cryptographic methods is therefore proposed to provide efficient data security while simultaneously addressing the problem of key distribution, thereby overcoming each scheme's security weaknesses [55].

Some previous studies have utilized asymmetric cryptography; for example, Purevjav [56] and Harba [57] employed Rivest Shamir Adleman (RSA), while N. Hong [58] and Xin [59] used Elliptic Curve Cryptography (ECC). Other studies have applied symmetric cryptography, i.e., Altigani [60], D'souza [61], Xin [59], and Harba [57] used Advanced Encryption Standard (AES), while Z. Hong [62] implemented the Data Encryption Standard (DES) in combination with Rivest Code 4 (RC4). Singh [63] similarly used symmetric encoding, while Purevjav [56] combined a public key encryption system with a symmetric hash function, thereby ensuring that messages encrypted with the public key could only be decrypted reasonably quickly using the private key. Harba [57] proposed a method of protecting data transfer using a hybrid technique: to ensure secure transmission, a symmetric AES algorithm was used to encrypt files; an asymmetric RSA algorithm was used to encrypt AES passwords; HMAC was used to encrypt passwords and symmetric data.

N. Hong [58] used the ECC password algorithm and the SM2 handshake agreement to solve security problems in the information transmission process, but failed to conduct a performance evaluation. Xin [59] proposed a mixed approach to encryption, integrating MD5 with ECC and AES, but again failed to evaluate performance results. Altigani [60] proposed combining AES with the Word Shift Coding Protocol steganography protocol, producing a model that improved the confidentiality of messages and overall system security. D'souza [61] proposed a hybrid approach, combining Dynamic Key Generation and Dynamic S-box Generation with an AES algorithm. This method used Dynamic Key Generation to add data complexity, thereby increasing confusion and diffusion in the ciphertext.

Z. Hong [62] offered a hybrid crypto algorithm that used the DES and RC4 encryption algorithms to encrypt communication data, but did not perform a performance evaluation. Singh [63] proposed a hybrid encryption scheme that made it difficult for attackers to learn information from messages sent through insecure data transmissions. Table 1 provides a comparison of several approaches to securing data transmission offered by previous studies, including the method provided by this study. All of these studies aim to secure transmission data using hybrid cryptography and generate multiple keys to increase security. Likewise, although almost all of these studies provide a layered or graded approach to security, few provided a security analysis.

Peer] Comput. Sci. reviewing PDF | (CS-2021:03:59198:1:0:NEW 5 Aug 2021) 
196 3. Materials \& Methods

197 3.1. The BRC4 Super Encryption Model

198 This study introduces BRC4 (Beaufort RC4) super encryption, a combination of Beaufort and 199 RC4 encryption. Beaufort encryption converts plain text to a poly-alphabetic substitution code 200 based on the Beaufort table, and although this algorithm is simple in its calculation processes, it 201 still generates secure random numbers [64]. RC4, meanwhile, is a stream cipher with a variable202 length key algorithm, which improves the confidentiality, randomness, and security of key 203 streams [65].

204 This research was conducted by simulating BRC4 super encryption on PLC program data (in the 205 instruction list [IL] format) from industrial machines. This simulation is used to anticipate MITM attacks; in other words, BRC4 super encryption (a combination of Beaufort and RC4 encryption)

207 is used to increase security and avoid MITM attacks (see Fig. 1).

208

209 The BRC4 super-encryption model consists of two models: an encryption model, i.e. a

210

211

212

213

214

215

216

217

218

219

220

221

222

223

224

225

226

227

228

229

230

231

232

233

234 combination of the Beaufort and the RC4 encryption processes (see Fig. 2), and a decryption model, i.e. a variety of RC4 and Beaufort decryption processes (see Fig. 3). The encryption model is installed in the data transmission section (RTU), while the decryption model is established in the data receiving section (MTU). This simulation model is built and tested using hardware with the following specifications: i7-6500U processor, 16GB RAM, and Windows 10 operating system (64-bit).

\subsection{Encryption Model Design}

Simulation is performed using Instruction List (IL) data from the Programmable Logic Controller (PLC) of several industrial machines. This IL data is in the form of an input-or-output logic command. Several IL data are used, with the following specifications: IL1 $=188$ lines, IL2 $=881$ lines, and IL3 = 4,571 lines. Each line consists of approximately 15 characters; as such, IL1 consists of 1,086 characters, IL2 consists of 7,158 characters, and IL3 consists of 33,046 characters.

\subsubsection{Data Reading}

The plaintext data are in the form of IL, i.e. a PLC program containing lists of sequence logic instructions in the form of sequentially executed text. In this paper, the data used is in the IL1 format.

\subsubsection{Converting to one-line format}

The plaintext data (IL1), presented in 188 lines and 1 column, is converted into a one-line array. To separate the lines, a semicolon (;) is used. This produces the following:

LD M8002;SET S0;STL S0;ZRST S20 S80;RST M0;OUT T0 K1;LD T0;ANI X001;ANI X012;MPS;ANI X015;SET S20;MPP;AND X015;SET S25;SET S30;STL S20;LD X013;ANI 
235

236

237

238

239

240

241

242

243

244

245

246

247

248

249

250

251

252

253

254

255

256

257

258

259

260

261

262

263

264

265

266

267

268

269

270

271

272

273

X002;OUT Y006;LD X014;ANI X003;OUT Y007;LD X015;OR X001;SET S0;LD X012;SET S90;RST S20;STL S90;LD M8013; ....etc.

Next, it is necessary to convert data from string format to numeric format to perform mathematical operations.

\subsubsection{Initial key generation}

This process aims to generate a random initial key, one that cannot easily be detected by attackers. Random initial key generation is conducted during each data transmission process.

Generation is carried out in the range of 1 to 256 bytes, with the length of the key depending on data size $(n)$ and character length of the initial key ( $p k$, i.e. $64,128,256,512,1,024,2,048$ bits); the longer the initial key, the more secure.

Figure 4 shows that, if the length of the plaintext $(H)$ is less than or equal to the size of the key, the length of the key will equal the length of the data $(n)$. Otherwise, if the length of the data is greater than the length of the key, then the length of the key is equal to the length of the chosen initial key $(p k, 256)$.

\subsubsection{Generation of Beaufort Encryption Key}

In Beaufort key generation, if the initial key is shorter than plaintext, the initial key is generated repeatedly along with the plaintext. Such generation uses a keystream generator approach, following equation (1).

$$
k_{i}=\left(k_{i-m}+k_{i-1}\right) \bmod 256
$$

$k_{i}$ is the key number- $i, k_{i-m}$ is the key number-i subtracted by the initial key $(m)$, and key number-i subtracted by 1 . The initial key length is 256 characters. Since the plaintext is 1,086 characters long, a Beaufort key is generated to be equal in length to the plaintext. Key numbers 257 to 1,086 are generated based on equation (1). For example:

$$
\begin{aligned}
& k_{257}=\left(k_{1}+k_{256}\right) \bmod 256 \\
& k_{257}=(206+174) \bmod 256 \\
& k_{257}=380 \bmod 256 \\
& k_{257}=124
\end{aligned}
$$

Figure 5 explains the process through which the Beaufort encryption key is generated. This process produces the Beaufort key for encryption, with the first 256 characters equal to the initial key and the generated results used for keys 257 to 1,086 .

\subsubsection{Beaufort Encryption}

Beaufort encryption uses the IL numeric format as plaintext and the Beaufort key. It is conducted using the following equation:

$$
\begin{aligned}
& E_{k}\left(P_{1}, P_{2}, \ldots, P_{m}\right)=\left(K_{1}-P_{1}, K_{2}-P_{2}, \ldots, K_{m}-P_{m}\right) \bmod 256 \\
& D_{k}\left(C_{1}, C_{2}, ., C_{m}\right)=\left(K_{1}-C_{1}, K_{2}-C_{2}, \ldots, K_{m}-C_{m}\right) \bmod 256
\end{aligned}
$$


274 where $E$ (encryption), $D$ (decryption), $P$ (plain text), $C$ (cipher), $K$ (key). The encryption process

275 begins with reading the plaintext $(n)$, namely the IL numeric format. A subtraction operation is

276 then carried out on each plaintext character, using a Beaufort key with a base 256 modulo,

277 thereby forming a Beaufort cipher (CB) is formed, as shown in Fig. 6.

278

279

280

281

\subsubsection{Generation of RC4 Encryption Key}

RC4 Key Generation consists of three steps. First, keystream generation is used to form the first

282 cipher block. Second, permutation generation is conducted with a key scheduling algorithm

283 function. Third, a pseudo-random number generation algorithm is used.

284 A key scheduling algorithm is used to initialize the permutations of array $S$. Key length is

285 defined as the number of bytes contained within a key, that is, between 1 and 256. The $S$ array is

286

287

288

289

290

291

292

293

294 initialized to the permutation identity; the $S$ array is processed to 256 iterations. After retrieving the random $S$ array, it is re-initialized, with the values of $i$ and $j$ being zero.

The PRGA process subsequently generates an RC4 key, by incrementing $i$, adding the values $S[i]$ and $S[j]$, and swapping two values. An $S$ value with an index equal to the numeric value $S[i]$, and $S[j]$, modulo 256, yields the RC4 key (see Fig. 7).

\subsubsection{RC4 Encryption}

RC4 encryption begins by reading the Beaufort cipher (CB) as plain text (Fig. 8). The system checks the plaintext $(n)$; if $n<256, \mathrm{RC} 4$ encryption is carried out against plain text $n$. Otherwise, blocks will be created using $(n / 256)$, rounded up and stored in STL.

295 Subsequently, $n$ is checked again, if $n<256$, the RC4 encryption process is conducted along with 296 the plaintext characters $(n)$. However, if $n>256$, the permutation process in the next block forms 297

298

299

300

301

302

303 the following array: $S[i]$ and $S[j]$. The values are then exchanged.

\subsubsection{The insertion of the initial key}

This section describes the process of inserting the key and key information by including them behind the cipher through a combination of encryption processes. The key entered is the initial key, produced by random generation, and the key information is the length of the preselected key. The key and key information are used to perform decryption when the password data has 304

305

306

307

308

309

310

311

312

313 been received. A randomly generated initial key is used to avoid MITM attacks.

While an attacker was to obtain ciphertext data, a combination of the cipher, initial key, and key information, said attacker would not obtain any information, as the ciphertext is random. If the attacker manages to separate the initial key from the ciphertext, the attacker will still not be able to read the cipher. The initial key is a different character length than the decryption key and can only be used after three generations: keystream generation, Beaufort decryption generation, and RC4 decryption generation.

\subsection{Decryption Model Design}

\subsubsection{Initial Key Separation}

Peer) Comput. Sci. reviewing PDF | (CS-2021:03:59198:1:0:NEW 5 Aug 2021) 
314 After the ciphertext is received, it is separated into three components: the cipher, the initial key, 315 and the key information (containing the key character length). The separation process is shown 316 in Fig. 9.

317

\subsubsection{Beaufort Decryption Key Generation}

320

321

322

323

324

325

326

327

328

329

330

331

332

333

334

335

336

337

338

339

340

341

342

343

344

345

346

347

348

349

350

351

352

353

This section discusses the generation of Beaufort's decryption keys, which follow the process illustrated in Fig. 5. First, the system calculates the cipher data $(n)$ and initial key $(m)$. If $n$ is more than $m$, one is added to the variable $i$; otherwise, $m$ is kept in variable $i$, and the original key is saved as the Beaufort key. If the length of $n$ is greater than $i$, keystream generation is performed.

\subsubsection{RC4 Decryption Key Generation}

After generating the Beaufort decryption key, the RC4 key is produced through several stages:

Keystream Generation (KG), Key Scheduling Algorithm (KSA) generation, and pseudo-random generation algorithm (PRGA).

Keystream generation aims to form the first block array and obtain a block length of up to 256 characters. A random block array key scheduling algorithm is generated based on the previous key (i.e. the Beaufort decryption key). Finally, the RC4 decryption key is obtained; this key is used for the final decryption process. In its process, the generation of the RC4 decryption key resembles the generation of the RC4 encryption key. This approach is described in Fig. 7.

\subsubsection{RC4 Decryption}

RC4 decryption is conducted, using the RC4 key, to separate the cipher data. The RC4 decryption process is described in Fig. 10. This process begins with the reading of the number of cipher characters. The subsequent processes through which keystreams and key scheduling algorithms are generated are intended to randomize the block array position. This is followed by generating a pseudo-random algorithm to obtain the RC4 key.

Therefore, if $n<256$, the RC4 decryption process will produce the Beaufort cipher (CB). If $n>256$, a block is formed to repeat the permutation of each character in the key array and $\mathrm{S}$ array, as well as to swap the values of $S[i]$ and $S[j]$. As a result, RC4 decryption (i.e. the Beaufort cipher) is obtained.

\subsubsection{Beaufort Decryption}

This section describes Beaufort decryption, with the Beaufort cipher (CB) used as input, using the Beaufort Key (KB). The detailed process is shown in Fig. 11.

This process begins by calculating the length of the CB, as stored in variable $n$. The character length of the initial key is stored in variable $m$. If the length of the ciphertext is greater than the length of the initial key $(n>m)$, the initial key is reproduced until the lengths are equal. The decryption process is done by adding each key character to $\mathrm{CB}$, then creating an array to store the results of decryption. This process produces the plaintext (New_IL) in numeric format. 
354

355

356

357

358

359

360

361

362

363

364

365

366

367

368

369

370

371

372

373

374

375

376

377

378

379

380

381

382

383

384

385

386

387

388

389

390

391

\subsubsection{Design of The Final Model}

This stage converts the numeric-type plaintext to the string type. It then changes the original oneline plain text format, and separator (;), into the original multi-line format. The process is detailed in Fig. 12.

\section{Results and Discussion}

This process reproduces IL data from the BRC4 super encryption system, which aims to increase the security of data transmission in SCADA systems via the DNP3 protocol. Its product is equivalent to the data sent via DNP3. As such, data can successfully be encrypted and decrypted using the BRC4 super-encryption method.

\subsection{Keyspace Analysis}

Keyspace analysis involves the analysis of keyspace within which the encryption system secures cipher data from brute force attacks. Brute-force attacks work by counting every possible combination that can form a password, and then testing them to determine the correct password. As the lengths and combinations of passwords grow, the amount of time it takes to find the correct password increases exponentially. Encryption systems must have very large keyspaces (greater than $2^{100}$ bits) to render brute force attacks ineffective [66], [67].

The proposed model consists of several key generators. The initial key generator, randomly created for each session, has key lengths of 64, 128, 256, 512, 1,024, and 2,048 bits. Each initial key character is generated from 256 bytes ASCII code, and thus has a spread value of 1 to 256 . A random initial key with a length of 16 characters (256 bits), using 256-byte ASCII code, would have a keyspace of $256^{16}$, equivalent to $2^{128}$. As such, an initial key that is 16 characters in length would be secure from brute force attacks, as would longer keys (i.e. 256, 512, 1,024, and 2,048 bits), as shown in Table 2 [68]. Table 2 shows that a key 64 bits in length would not be secure from brute force attacks; a minimum key size of 128 bits is necessary to guarantee a secure encryption process.

\subsection{Correlation Coefficient Analysis}

Correlation coefficient analysis aims to determine the correlation between plaintext and ciphertext data. If the correlation value is equal to 1 , it means that the two data are the same. Conversely, if the correlation value is lower than one (or close to 0 ), the two data are different; there is thus no relationship, and increased randomness (see Table 3). The less related the text, the better, as increased randomness means increased difficulty deciphering the relationship between plain text and encoded text [67], [69]. The correlation between plaintext and ciphertext data is formulated as:

$$
r=\frac{n\left(\sum x y\right)-\left(\sum x\right)\left(\sum y\right)}{\sqrt{\left[n \sum x^{2}-\left(\sum x\right)^{2}\right]\left[n \sum y^{2}-\left(\sum y\right)^{2}\right]}}
$$


392 In this formula, $r$ is the correlation value, $x$ is the plaintext data, and $y$ is the ciphertext data.

393 Based on equation (4), it can be seen that the proposed method produces a correlation value of 3940.010 for IL1 data, 0.006 for IL2 data, and 0.001 for IL3 data (see Table 4).

395 Referring to Pearson (Table 3), the correlation value for all three data may be categorized as "no 396 correlation," meaning that the plaintext and ciphertext data are not the same (i.e. uncorrelated).

397 Furthermore, the correlation value for the three data is closer to zero than the correlation value

398 for data encrypted using the Beaufort cipher or RC4 in isolation. This shows that BRC4 super399 encryption can improve the security of data transmission.

400

401

402

403

404

405

406

407

408

409

410

411

412

413

414

415

416

417

418

419

420

421

422

423

424

425

426

427

428

429

430

431

\subsection{Information Entropy Analysis}

In cryptographic theory, information entropy is defined as a measure of the randomness of the amount of information in a message. Entropy is expressed in units of bits to express the degree of information randomness. Under random conditions, encrypted information with ciphertext data should have an optimum entropy value close to $\approx 8$; an entropy close to 8 , thus, indicates that an encryption system is designed to be secure from MITM attacks [70]. The entropy value may be determined using the following equation [71]:

$$
H=-\sum_{k=0}^{n} P(k) \log _{2}(P(k))
$$

With $\mathrm{H}$ as the entropy value, $\mathrm{n}$ is the number of different symbols or codes in a message, and $\mathrm{P}(\mathrm{k})$ is the probability of symbol occurrence in the ciphertext.

Using the proposed method, entropy values of 7.84 (for IL1), 7.98 (for IL2), and 7.99 (for IL3) were returned. The proposed model's IL data has an entropy value close to 8 , which indicates a high degree of randomness, and thus the ciphertext is secure from MITM attacks. A comparison of the entropy values for data encrypted using the Beaufort, RC4, and BRC4 models is provided in Table 5. Data encrypted through BRC4 super-encryption is closest to 8.00 in value, which indicates that $\mathrm{BRC} 4$ super-encryption is more secure than Beaufort or RC4 encryption alone.

\subsection{Visual analysis}

Visual analysis aims to measure the results of IL data encryption using a histogram and compare the distribution of plaintext and ciphertext data. When a ciphertext histogram is more diverse and differently distributed than the plaintext histogram, it can be concluded that the ciphertext does not provide any clues or information that can be deciphered by MITM attacks.

Figure 13 shows the histogram results for IL data (the first 500 of 33,046 characters). It notes that data distribution between plaintext and ciphertext is very varied, and thus ciphertext data is secure from MITM attacks. The plaintext is distributed in the numeric range of 10 to 99 , while the ciphertext has a distribution of 1 to 256 . As such, the ciphertext is more secure from MITM attacks than texts encrypted using Beaufort or RC4 in isolation.

\subsection{Time Complexity Analysis}

Time complexity, or $\mathrm{T}(\mathrm{n})$, is measured based on the number of computational steps required to run the algorithm as a function of the input size (n). Calculations are based on multiple operator 
432 steps, procedures/functions, control steps, and loops [67]. For compatibility, the symbols $\mathrm{Ci}$ and $433 \sum$ are used for calculation, as follows: $\mathrm{C} 1$ is used to symbolize the assignment; $\mathrm{C} 2$ is used to 434 symbolize the number of arithmetic operators used; C3 is used to symbolize built-in 435 procedures/functions such as input, output, or user-defined procedures/functions; $\mathrm{C} 4$ is used to 436 symbolize the loop operation; and C5 is used to symbolize the structure of the branching 437 conditions. Finally, $\sum$ is used to represent the number of steps involved in each Ci symbol.

438 The results of the time complexity calculation for the encryption algorithm (see Appendix 1) are 439 as follows:

440

441

442

443

444

$T(n)=(12+2 n+4 n / 256) C 1+(5652+35 n+7 n / 256) C 2+(11+n / 256) C 3+(1023+7 n+n / 256) C 4+$ $(3+\mathrm{n} / 256) \mathrm{C} 5$. $=(12 \mathrm{C} 1+5652 \mathrm{C} 2+11 \mathrm{C} 3+1023 \mathrm{C} 4+3 \mathrm{C} 5)+(516 \mathrm{C} 1 / 256+8967 \mathrm{C} 2 / 256+\mathrm{nC} 3 / 256+$

445

446

447

448

449

450

451

452

453

454

455

456

457

458 $1793 \mathrm{C} 4 / 256+\mathrm{C} 5 / 256) \mathrm{n}$.

$\mathrm{T}(\mathrm{n}) \approx \mathrm{O}(\mathrm{n})$.

Meanwhile, the results of the time complexity calculation (see Appendix 2) for the decryption algorithm are as follows:

$$
\begin{aligned}
T(n)= & (268+515 n+3 n / 256) C 1+(8971+291 n+4 n / 256) C 2+(9+3 n+n / 256) C 3+ \\
& (1024+264 n+n / 256) C 4+(3+n / 256) C 5 . \\
= & (268 C 1+8971 C 2+9 C 3+1024 C 4+3 C 5)+(131,843 C 1 / 256+74,500 C 2 / 256+ \\
& 769 C 3 / 256+67,585 C 4 / 256+C 5 / 256) n .
\end{aligned}
$$

$\mathrm{T}(\mathrm{n}) \approx \mathrm{O}(\mathrm{n})$.

This analysis shows that the encryption and decryption algorithms may be categorized as having linear complexity, meaning that processing time corresponds positively and linearly with data size. In other words, if the algorithm requires $n$ steps to handle data of $n$ size, it will need $2 n$ steps for data of $2 \mathrm{n}$ size.

460

461

462

\subsection{Cryptanalysis Solutions}

Super encryption BRC4 is a proposed method that combines the Beaufort and RC4 ciphers,

464 wherein four symmetric key generators are generated dynamically every session. We must ascertain whether this proposed method can overcome the weaknesses of the Beaufort and RC4 ciphers in isolation. According to Hughes [72], the Beaufort cipher - as with the Vigenere cipher-has several weaknesses. In their case, Hughes used neither the Vigenère nor the Vernam ciphers, as both needed to meet the same three requirements to comply with Shannon's definition of complete secrecy. According to Alallayah [73], the Vigenere cipher offers a combination of lowercase alphabetical characters, with a maximum length of $676\left(26^{*} 26\right)$ bytes. 
472 with an initialization vector (IV) modifier for both the 24 and 128-bit modifiers (which are

473 known to encrypt different messages). All of these weaknesses have been anticipated by the

474 proposed method, as shown in Tables 6 and 7.

475

476

477

\section{Conclusions}

478

479

480

481

482

483

484

485

486

487

488

489

490

491

492

493

494

495

496

497

498

499

500

501

502

503

504

505

506

507

508

509

510

511

512

\section{Acknowledgments}

This research is supported by doctoral dissertation research from the Directorate General of Higher Education, the Indonesian Ministry of Education and Culture through contract number 2084/UN1/DITLIT/DIT-LIT/PT/2020. We also thank the Nuclear Energy Regulatory Agency (BAPETEN) and Universitas Gadjah Mada for their support.

\section{References}

[1] P. Jain and P. Tripathi, "SCADA security: a review and enhancement for DNP3 based systems," CSI Trans. ICT, vol. 1, no. 4, pp. 301-308, Dec. 2013, doi: 10.1007/s40012013-0024-2.

[2] R. Czechowski, P. Wicher, and B. Wiecha, "Cyber security in communication of SCADA systems using IEC 61850," in 2015 Modern Electric Power Systems (MEPS), Jul. 2015, pp. 1-7, doi: 10.1109/MEPS.2015.7477223.

[3] H. Shabani and M. Ahmed, "Novel IEEE 802. 15.4 protocol for modern SCADA communication systems," 2014 IEEE 8th Int. Power Eng. Optim. Conf., no. March, pp. 597-601, 2014, [Online]. Available:

http://ieeexplore.ieee.org/xpls/abs_all.jsp?arnumber=6814498. 
513 [4] A. Rezai, P. Keshavarzi, and Z. Moravej, "Key management issue in SCADA networks: A

514

515

516

517

518

519

520

521

522

523

524

525

526

527

528

529

530

531

532

533

534

535

536

537

538

539

540

541

542

543

544

545

546

547

548

549

550

551

552

553

554

555

556

557

558 review," Eng. Sci. Technol. an Int. J., vol. 20, no. 1, pp. 354-363, 2017, doi:

10.1016/j.jestch.2016.08.011.

[5] A. R. Mathew, "Cyber-infrastructure connections and smart gird security," Int. J. Eng. Adv. Technol., vol. 8, no. 6, pp. 2285-2287, 2019, doi: 10.35940/ijeat.F8681.088619.

[6] Y. Prayudi, A. Ashari, and T. K Priyambodo, "A Proposed Digital Forensics Business Model to Support Cybercrime Investigation in Indonesia," Int. J. Comput. Netw. Inf. Secur., vol. 7, no. 11, pp. 1-8, 2015, doi: 10.5815/ijcnis.2015.11.01.

[7] B. Kesler, "The Vulnerability of Nuclear Facilities to Cyber Attack," Strateg. Insights, vol. 10, no. 1, pp. 15-25, 2011.

[8] P. H. Nguyen, S. Ali, and T. Yue, "Model-based security engineering for cyber-physical systems: A systematic mapping study," Inf. Softw. Technol., vol. 83, pp. 116-135, 2017, doi: 10.1016/j.infsof.2016.11.004.

[9] H. Orojloo and M. A. Azgomi, "A method for evaluating the consequence propagation of security attacks in cyber-physical systems," Futur. Gener. Comput. Syst., vol. 67, pp. 5771, 2017, doi: 10.1016/j.future.2016.07.016.

[10] D. Dragomir, L. Gheorghe, S. Costea, and A. Radovici, "A Survey on Secure Communication Protocols for IoT Systems," in 2016 International Workshop on Secure Internet of Things (SIoT), 2016, pp. 47-62, doi: 10.1109/SIoT.2016.012.

[11] B. Chen, N. Pattanaik, A. Goulart, K. L. Butler-Purry, and D. Kundur, "Implementing attacks for modbus/TCP protocol in a real-time cyber physical system test bed," 2015, doi: 10.1109/CQR.2015.7129084.

[12] C. Cremers, M. Dehnel-wild, and K. Milner, Secure authentication in the grid: A formal analysis of DNP3: SAv5, vol. 10492 LNCS, no. June. 2017, pp. 389-407.

[13] J. T. Robinson et al., "Development of the Intercontrol Center Communications Protocol (ICCP) [power system control]," Proc. Power Ind. Comput. Appl. Conf., pp. 449-455, 1995, doi: 10.1109/PICA.1995.515277.

[14] S. Bratus et al., "Implementing a vertically hardened DNP3 control stack for power applications," Proc. 2nd Annu. Ind. Control Syst. Secur. Work. - ICSS '16, pp. 45-53, 2016, doi: 10.1145/3018981.3018985.

[15] I. A. I. A. Siddavatam and F. Kazi, "Security assessment framework for cyber physical systems: A case-study of DNP3 protocol," 2015 IEEE Bombay Sect. Symp. Front. Technol. Fuelling Prosper. Planet People, IBSS 2015, 2016, doi: 10.1109/IBSS.2015.7456631.

[16] D. Dahal, Ujjwal; Cheten, "Substation Automation: Matlab and OPC Driven Substation Monitoring System," 2011.

[17] G. Kapil, A. Agrawal, A. Attaallah, A. Algarni, R. Kumar, and R. A. Khan, "Attribute based honey encryption algorithm for securing big data: Hadoop distributed file system perspective," PeerJ Comput. Sci., vol. 2020, no. 2, pp. 1-31, 2020, doi: 10.7717/peerjcs.259.

[18] I. Shin, D. Eom, and B. Song, "The CoAP-based M2M gateway for distribution automation system using DNP3.0 in smart grid environment," in 2015 IEEE International Conference on Smart Grid Communications (SmartGridComm), Nov. 2015, pp. 713-718, doi: 10.1109/SmartGridComm.2015.7436385.

[19] M. Kim, W. Jo, J. Kim, and T. Shon, "Visualization for internet of things : power system and financial network cases," Multimed. Tools Appl., 2018, doi: 10.1007/s11042-018- 
559

560

561

562

563

564

565

566

567

568

569

570

571

572

573

574

575

576

577

578

579

580

581

582

583

584

585

586

587

588

589

590

591

592

593

594

595

596

597

598

599

600

601

602

603

604

6730-x.

[20] M. Faisal, A. A. Cardenas, and A. Wool, "Modeling Modbus TCP for intrusion detection," 2016 IEEE Conf. Commun. Netw. Secur. CNS 2016, pp. 386-390, 2017, doi: 10.1109/CNS.2016.7860524.

[21] S. Senthivel, I. Ahmed, and V. Roussev, "SCADA network forensics of the PCCC protocol," Digit. Investig., vol. 22, pp. S57-S65, 2017, doi: 10.1016/j.diin.2017.06.012.

[22] M. Mantere, M. Sailio, and S. Noponen, "Network Traffic Features for Anomaly Detection in Specific Industrial Control System Network," Futur. Internet, vol. 5, no. 4, pp. 460-473, Sep. 2013, doi: 10.3390/fi5040460.

[23] D. Lee, H. Kim, K. Kim, and P. D. Yoo, "Simulated Attack on DNP3 Protocol in SCADA System," Inst. Electron. Inf. Commun. Eng., pp. 1-6, 2014, [Online]. Available: http://caislab.kaist.ac.kr/publication/paper_files/2014/SCIS2014_DS.pdf.

[24] J. Park, Y. Suh, and C. Park, "Implementation of cyber security for safety systems of nuclear facilities," Prog. Nucl. Energy, vol. 88, pp. 88-94, Apr. 2016, doi: 10.1016/j.pnucene.2015.12.009.

[25] T. Bartman and K. Carson, "Securing communications for SCADA and critical industrial systems," in 2016 69th Annual Conference for Protective Relay Engineers (CPRE), Apr. 2016, pp. 1-10, doi: 10.1109/CPRE.2016.7914914.

[26] S. Shitharth and D. P. Winston, "A novel IDS technique to detect DDoS and sniffers in smart grid," IEEE WCTFTR 2016 - Proc. 2016 World Conf. Futur. Trends Res. Innov. Soc. Welf., pp. 3-8, 2016, doi: 10.1109/STARTUP.2016.7583897.

[27] M. A. Rahman, A. H. M. Jakaria, and E. Al-Shaer, "Formal Analysis for Dependable Supervisory Control and Data Acquisition in Smart Grids," 2016 46th Annu. IEEE/IFIP Int. Conf. Dependable Syst. Networks, pp. 263-274, 2016, doi: 10.1109/DSN.2016.32.

[28] I. Sembiring, "Implementation of honeypot to detect and prevent distributed denial of service attack," Proc. - 2016 3rd Int. Conf. Inf. Technol. Comput. Electr. Eng. ICITACEE 2016, pp. 345-350, 2017, doi: 10.1109/ICITACEE.2016.7892469.

[29] R. Amoah, S. Camtepe, and E. Foo, "Formal modelling and analysis of DNP3 secure authentication," J. Netw. Comput. Appl., vol. 59, pp. 345-360, 2016, doi: 10.1016/j.jnca.2015.05.015.

[30] S. Smaiah, A. Khellaf, and T. Cherifi, "The implementation of SCADA open protocol IEC60870-5-101 on ARDUINO UNO board," 2015 4th Int. Conf. Electr. Eng. ICEE 2015, 2016, doi: 10.1109/INTEE.2015.7416844.

[31] M. Friesen, "Evaluation of multipath communication protocols for highly reliable cyberphysical systems," 2016.

[32] N. Moreira, E. Molina, J. Lázaro, E. Jacob, and A. Astarloa, "Cyber-security in substation automation systems," Renew. Sustain. Energy Rev., vol. 54, pp. 1552-1562, Feb. 2016, doi: 10.1016/j.rser.2015.10.124.

[33] I. Darwish, O. Igbe, and T. Saadawi, "Experimental and theoretical modeling of DNP3 attacks in smart grids," 2015 36th IEEE Sarnoff Symp., pp. 155-160, 2015, doi: 10.1109/SARNOF.2015.7324661.

[34] R. Amoah, "Formal Security Analysis of the DNP3-Secure Authentication Protocol," no. 2016, p. 164, 2016, [Online]. Available: http://eprints.qut.edu.au/93798/1/Raphael_Amoah_Thesis.pdf.

[35] B. Chen, K. L. Butler-Purry, A. Goulart, and D. Kundur, "Implementing a real-time cyberphysical system test bed in RTDS and OPNET," 2014 North Am. Power Symp. NAPS 
605

606

607

608

609

610

611

612

613

614

615

616

617

618

619

620

621

622

623

624

625

626

627

628

629

630

631

632

633

634

635

636

637

638

639

640

641

642

643

644

645

646

647

648

649

650

2014, pp. 1-6, 2014, doi: 10.1109/NAPS.2014.6965381.

[36] J. Nivethan and M. Papa, "A Linux-based firewall for the DNP3 protocol," in 2016 IEEE Symposium on Technologies for Homeland Security (HST), May 2016, pp. 1-5, doi: 10.1109/THS.2016.7568963.

[37] C. Singh, A. Nivangune, and P. Mrinal, "Function Code Based Vulnerability Analysis of DNP3," 2016.

[38] W. Ahn, M. Chung, B. G. Min, and J. Seo, "Development of Cyber-Attack Scenarios for Nuclear Power Plants Using Scenario Graphs," Int. J. Distrib. Sens. Networks, vol. 2015, 2015, doi: 10.1155/2015/836258.

[39] A. Hou, C. Hu, K. Ma, Z. Cai, C. Huang, and T. Pan, "Research on modeling and simulation of communication in power SCADA system," Proc. 5th IEEE Int. Conf. Electr. Util. Deregulation, Restruct. Power Technol. DRPT 2015, pp. 226-230, 2016, doi: 10.1109/DRPT.2015.7432232.

[40] A. Shahzad, S. Musa, and M. Irfan, "N-Secure Cryptography Solution for SCADA Security Enhancement," Trends Appl. Sci. Res., 2014, doi: 10.3923/tasr.2014.381.395.

[41] B. Tare, S. Waghmare, I. Siddavatam, F. Kazi, and N. Singh, "Security analysis of DNP3 using CPN model with state space report representation using LDA," 2016 Indian Control Conf. ICC 2016 - Proc., no. Icc, pp. 25-31, 2016, doi:

10.1109/INDIANCC.2016.7441101.

[42] S. El Bouanani, O. Achbarou, M. A. Kiram, and A. Outchakoucht, "Towards Understanding Internet of Things Security and its Empirical Vulnerabilities : A Survey," Int. J. Adv. Comput. Sci. Appl., vol. 10, no. 10, pp. 337-345, 2019, [Online]. Available: https://thesai.org/Downloads/Volume10No10/Paper_48Towards_understanding_Internet_of_Things_Security.pdf.

[43] I. Darwish, O. Igbe, and T. Saadawi, "Vulnerability assessment and experimentation of smart grid DNP3," J. Cyber Secur. Mobil., vol. 5, no. 1, pp. 23-54, 2016, doi: 10.13052/jcsm2245-1439.513.

[44] M. A. M. Isa, M. M. Ahmad, N. F. M. Sani, H. Hashim, and R. Mahmod, "Cryptographic key exchange protocol with message authentication codes (MAC) using finite state machine," in Procedia Computer Science, 2014, vol. 42, no. C, pp. 263-270, doi: 10.1016/j.procs.2014.11.061.

[45] A. P. Premnath, J.-Y. Jo, and Y. Kim, "Application of NTRU Cryptographic Algorithm for SCADA Security," in 2014 11th International Conference on Information Technology: New Generations, Apr. 2014, pp. 341-346, doi: 10.1109/ITNG.2014.38.

[46] K. Atighehchi, T. Muntean, S. Parlanti, R. Rolland, and L. Vallet, "A Cryptographic Keys Transfer Protocol for Secure Communicating Systems," in 2010 12th International Symposium on Symbolic and Numeric Algorithms for Scientific Computing, Sep. 2010, pp. 339-343, doi: 10.1109/SYNASC.2010.56.

[47] P. H. Yu and U. W. Pooch, "A secure dynamic cryptographic and encryption protocol for wireless networks," in IEEE EUROCON 2009, May 2009, vol. 2, pp. 1860-1865, doi: 10.1109/EURCON.2009.5167898.

[48] N. Mohd, A. Singh, and H. S. Bhadauria, "Bioinspired immune system for intrusions detection system in self configurable networks," Int. J. Adv. Comput. Sci. Appl., vol. 10, no. 10, pp. 159-166, 2019.

[49] A. Shahzad et al., "The protocol design and New approach for SCADA security enhancement during sensors broadcasting system," Multimed. Tools Appl., vol. 75, no. 22,

Peer] Comput. Sci. reviewing PDF | (CS-2021:03:59198:1:0:NEW 5 Aug 2021) 
651

652

653

654

655

656

657

658

659

660

661

662

663

664

665

666

667

668

669

670

671

672

673

674

675

676

677

678

679

680

681

682

683

684

685

686

687

688

689

690

691

692

693

694

695

696

pp. 14641-14668, 2016, doi: 10.1007/s11042-015-3050-2.

[50] P. Singh, S. Garg, V. Kumar, and Z. Saquib, "A testbed for SCADA cyber security and intrusion detection," 2015, doi: 10.1109/SSIC.2015.7245683.

[51] P. Fremantle and P. Scott, "A survey of secure middleware for the Internet of Things," PeerJ Comput. Sci., vol. 3, no. 5, p. e114, May 2017, doi: 10.7717/peerj-cs. 114.

[52] S. K. H. Islam, R. Amin, G. P. Biswas, M. S. Farash, X. Li, and S. Kumari, "An improved three party authenticated key exchange protocol using hash function and elliptic curve cryptography for mobile-commerce environments," J. King Saud Univ. - Comput. Inf. Sci., vol. 29, no. 3, pp. 311-324, Jul. 2017, doi: 10.1016/j.jksuci.2015.08.002.

[53] S. Dey and R. Ghosh, "A Review of Cryptographic Properties of 4-Bit S-Boxes with Generation and Analysis of Crypto Secure S-Boxes," in Computer and Cyber Security, Auerbach Publications, 2018, pp. 527-555.

[54] N. N. Mohamed, Y. Mohd Yussof, M. A. Saleh, and H. Hashim, "Hybrid Cryptographic Approach For Internet Of Things Applications: A Review," J. Inf. Commun. Technol., vol. 19, no. 3, pp. 279-319, Jul. 2020, doi: 10.32890/jict2020.19.3.1.

[55] E. H. Riyadi, T. K. Priyambodo, and A. E. Putra, "The Dynamic Symmetric Four-KeyGenerators System for Securing Data Transmission in the Industrial Control System," Int. J. Intell. Eng. Syst., vol. 14, no. 1, pp. 376-386, Feb. 2021, doi: 10.22266/ijies2021.0228.35.

[56] S. Purevjav, T. Kim, and H. Lee, "Email encryption using hybrid cryptosystem based on Android," Int. Conf. Adv. Commun. Technol. ICACT, vol. 2016-March, pp. 426-429, 2016, doi: 10.1109/ICACT.2016.7423418.

[57] E. S. I. Harba, "Secure Data Encryption Through a Combination of AES, RSA and HMAC," Eng. Technol. Appl. Sci. Res., vol. 7, no. 4, pp. 1781-1785, Aug. 2017, doi: 10.48084/etasr.1272.

[58] N. Hong and Z. Xuefeng, "A security framework for internet of things based on SM2 cipher algorithm," Proc. - 2013 Int. Conf. Comput. Inf. Sci. ICCIS 2013, pp. 13-16, 2013, doi: 10.1109/ICCIS.2013.12.

[59] M. Xin, "A Mixed Encryption Algorithm Used in Internet of Things Security Transmission System," Proc. - 2015 Int. Conf. Cyber-Enabled Distrib. Comput. Knowl. Discov. CyberC 2015, pp. 62-65, 2015, doi: 10.1109/CyberC.2015.9.

[60] A. Altigani and B. Barry, "A hybrid approach to secure transmitted messages using advanced encryption standard (AES) and Word Shift Coding Protocol," Proc. - 2013 Int. Conf. Comput. Electr. Electron. Eng. 'Research Makes a Differ. ICCEEE 2013, pp. 134139, 2013, doi: 10.1109/ICCEEE.2013.6633920.

[61] F. J. D'souza and D. Panchal, "Advanced encryption standard (AES) security enhancement using hybrid approach," Proceeding - IEEE Int. Conf. Comput. Commun. Autom. ICCCA 2017, vol. 2017-Janua, pp. 647-652, 2017, doi: 10.1109/CCAA.2017.8229881.

[62] Z. Y. Hong, Z. P. Qiu, S. L. Zeng, S. De Wang, and M. Sandrine, "Research on fusion encryption algorithm for internet of things monitoring equipment," Proc. - 14th Int. Symp. Pervasive Syst. Algorithms Networks, I-SPAN 2017, 11th Int. Conf. Front. Comput. Sci. Technol. FCST 2017 3rd Int. Symp. Creat. Comput. ISCC 2017, vol. 2017-Novem, pp. 425-429, 2017, doi: 10.1109/ISPAN-FCST-ISCC.2017.49.

[63] R. Singh, I. Panchbhaiya, A. Pandey, and R. H. Goudar, "Hybrid Encryption Scheme (HES): An approach for transmitting secure data over internet," Procedia Comput. Sci., 
697

698

699

700

701

702

703

704

705

706

707

708

709

710

711

712

713

714

715

716

717

718

719

720

721

722

723

724

725

726

727

728

729

730

731

732

733

734 vol. 48, no. C, pp. 51-57, 2015, doi: 10.1016/j.procs.2015.04.109.

[64] R. N. Sari and R. S. Hayati, "Beaufort Cipher Algorithm Analysis Based on the Power Lock-Blum Blum Shub in Securing Data," in 2018 6th International Conference on Cyber and IT Service Management (CITSM), Aug. 2018, no. Citsm, pp. 1-4, doi: 10.1109/CITSM.2018.8674368.

[65] J. Zhang, H. Liu, and L. Ni, "A Secure Energy-Saving Communication and Encrypted Storage Model Based on RC4 for EHR," IEEE Access, vol. 8, pp. 38995-39012, 2020, doi: 10.1109/ACCESS.2020.2975208.

[66] M. Hamdi, R. Rhouma, and S. Belghith, "A selective compression-encryption of images based on SPIHT coding and Chirikov Standard Map," Signal Processing, vol. 131, pp. 514-526, 2017, doi: 10.1016/j.sigpro.2016.09.011.

[67] E. Setyaningsih, R. Wardoyo, and A. K. Sari, "Securing color image transmission using compression-encryption model with dynamic key generator and efficient symmetric key distribution," Digit. Commun. Networks, vol. 6, no. 4, pp. 486-503, Nov. 2020, doi: 10.1016/j.dcan.2020.02.001.

[68] E. H. Riyadi, T. K. Priyambodo, and A. E. Putra, "Real-time Testing on Improved Data Transmission Security in the Industrial Control System," in 2020 3rd International Seminar on Research of Information Technology and Intelligent Systems (ISRITI), Dec. 2020, pp. 129-134, doi: 10.1109/ISRITI51436.2020.9315339.

[69] E. Setyaningsih and R. Wardoyo, "Review of Image Compression and Encryption Techniques,” Int. J. Adv. Comput. Sci. Appl., vol. 8, no. 2, pp. 83-94, 2017, doi: 10.14569/IJACSA.2017.080212.

[70] E. Setyaningsih, R. Wardoyo, and A. K. Sari, "New Compression-Encryption Algorithm Using Chaos-Based Dynamic Session Key,” Int. J. Smart Sens. Intell. Syst., vol. 11, no. 1, pp. 1-19, Mar. 2018, doi: 10.21307/ijssis-2018-004.

[71] A. Shukla and S. Kumar, "Analysis of secure watermarking based on DWT-SVD technique for piracy," in 2016 International Conference on Computing, Communication and Automation (ICCCA), Apr. 2016, pp. 1110-1115, doi: 10.1109/CCAA.2016.7813882.

[72] J. M. Hughes, "Seven Steps to a Forever-Safe Cipher ( An Introduction to PolySubstitution Encryption ) Seven Steps to a Forever-Safe Cipher ( An Introduction to PolySubstitution Encryption )," no. June 2018, 2019.

[73] K. Alallayah, M. Amin, W. A. El-Wahed, and A. Alhamami, "Attack and construction of simulator for some of cipher systems using Neuro-Identifier," Int. Arab J. Inf. Technol., vol. 7, no. 4, pp. 365-372, 2010.

[74] S. Fluhrer, I. Mantin, and A. Shamir, "Weaknesses in the Key Scheduling Algorithm of RC4," in Lecture Notes in Computer Science (including subseries Lecture Notes in Artificial Intelligence and Lecture Notes in Bioinformatics), vol. 2259, 2001, pp. 1-24. 
Figure 1

The proposed research method

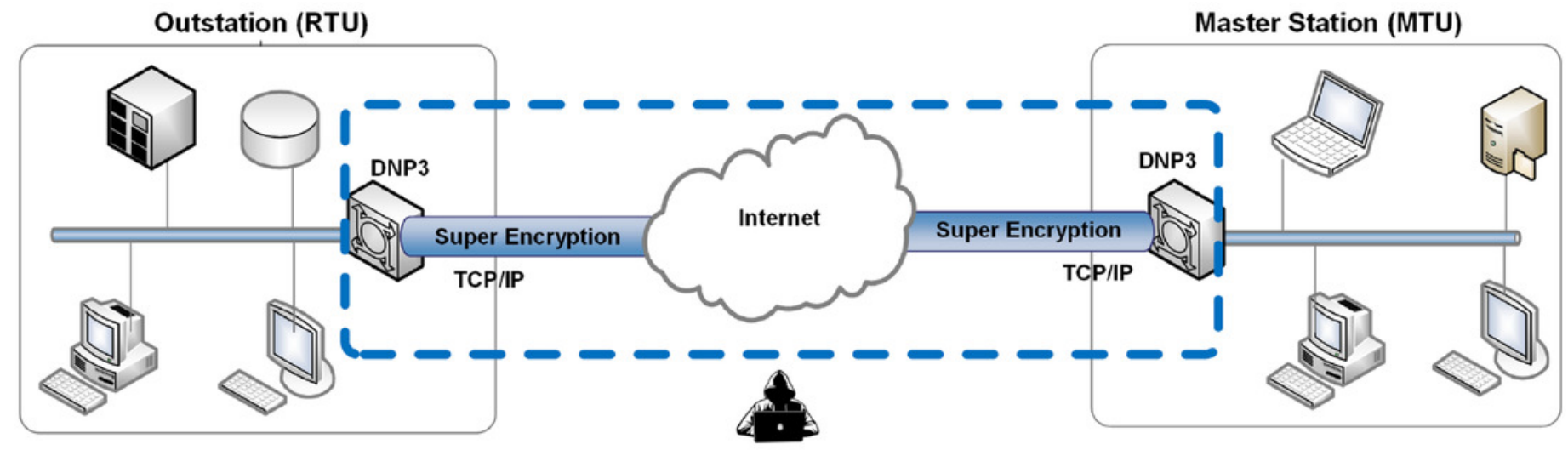


Figure 2

Encryption Model

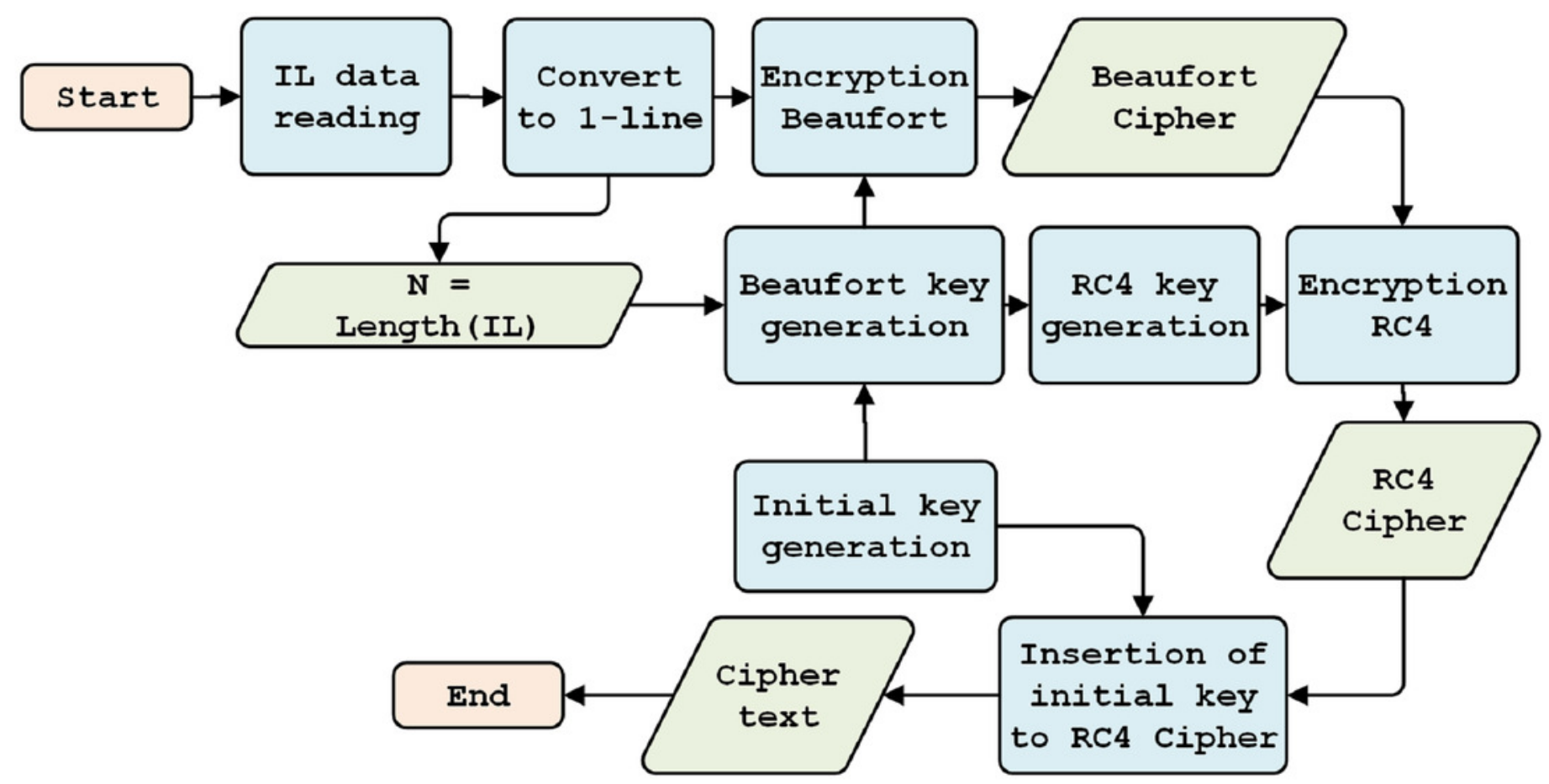


Figure 3

Decryption Model

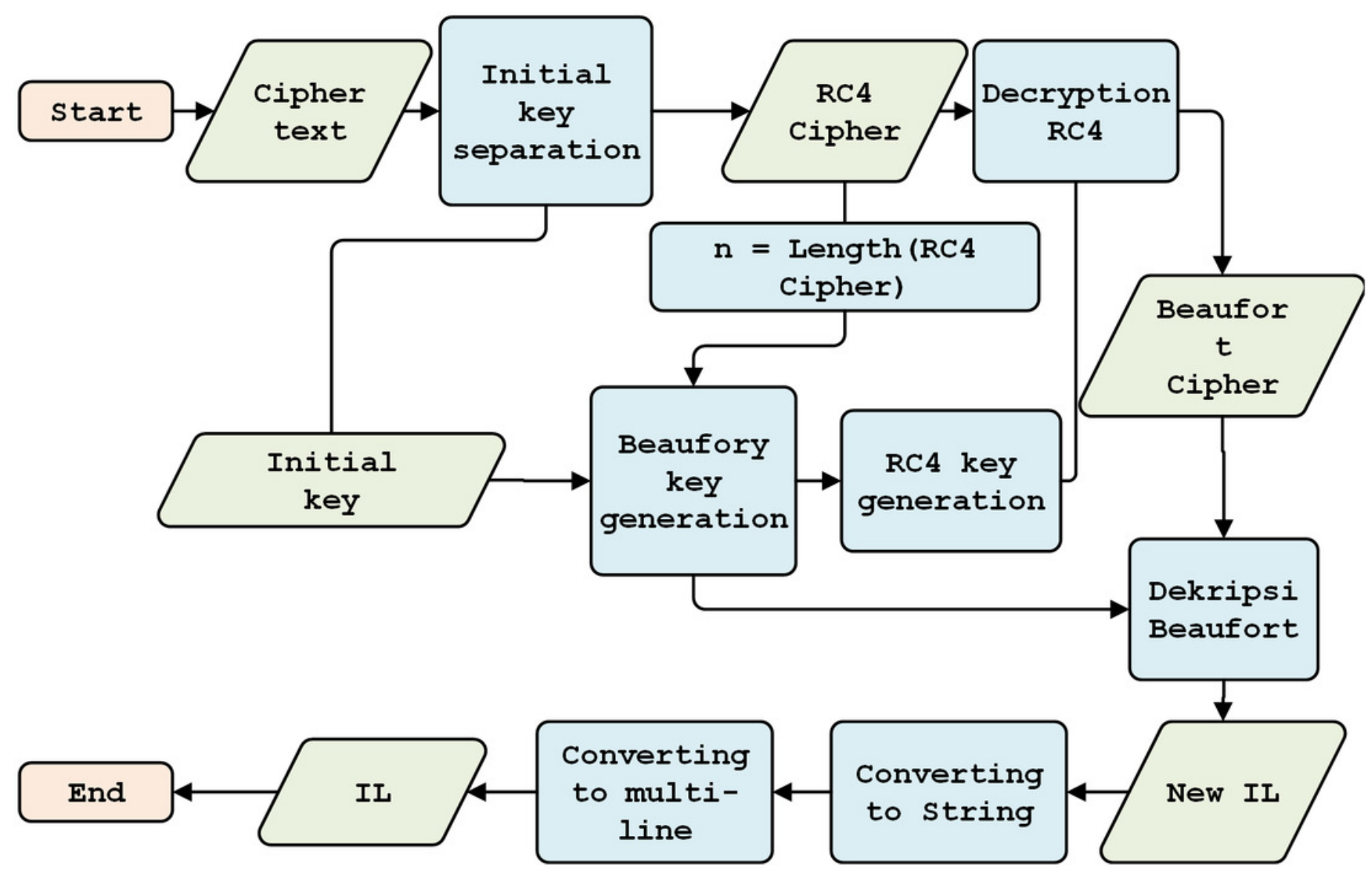


Figure 4

Initial key generation

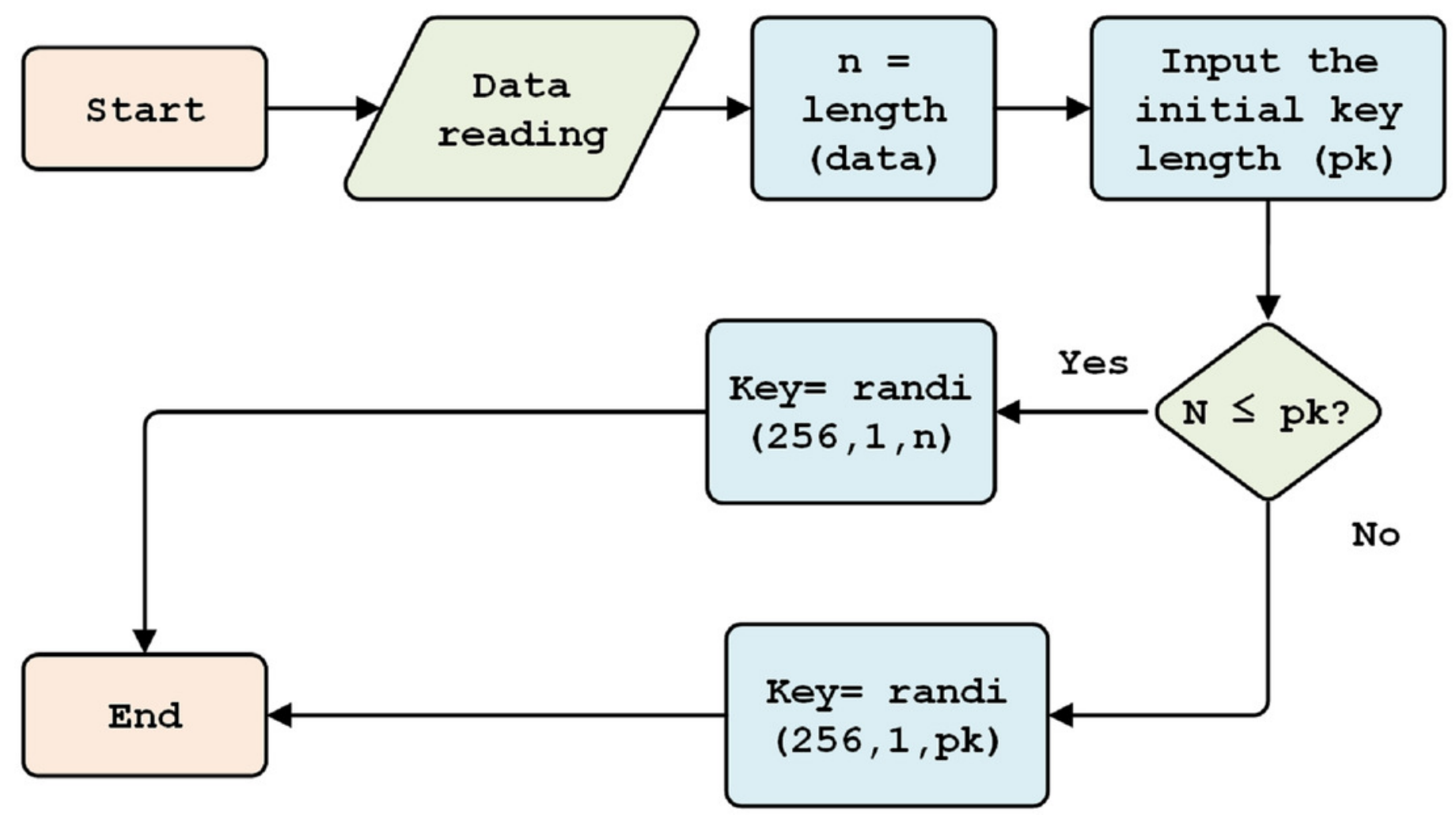


Figure 5

Beaufort Key generation

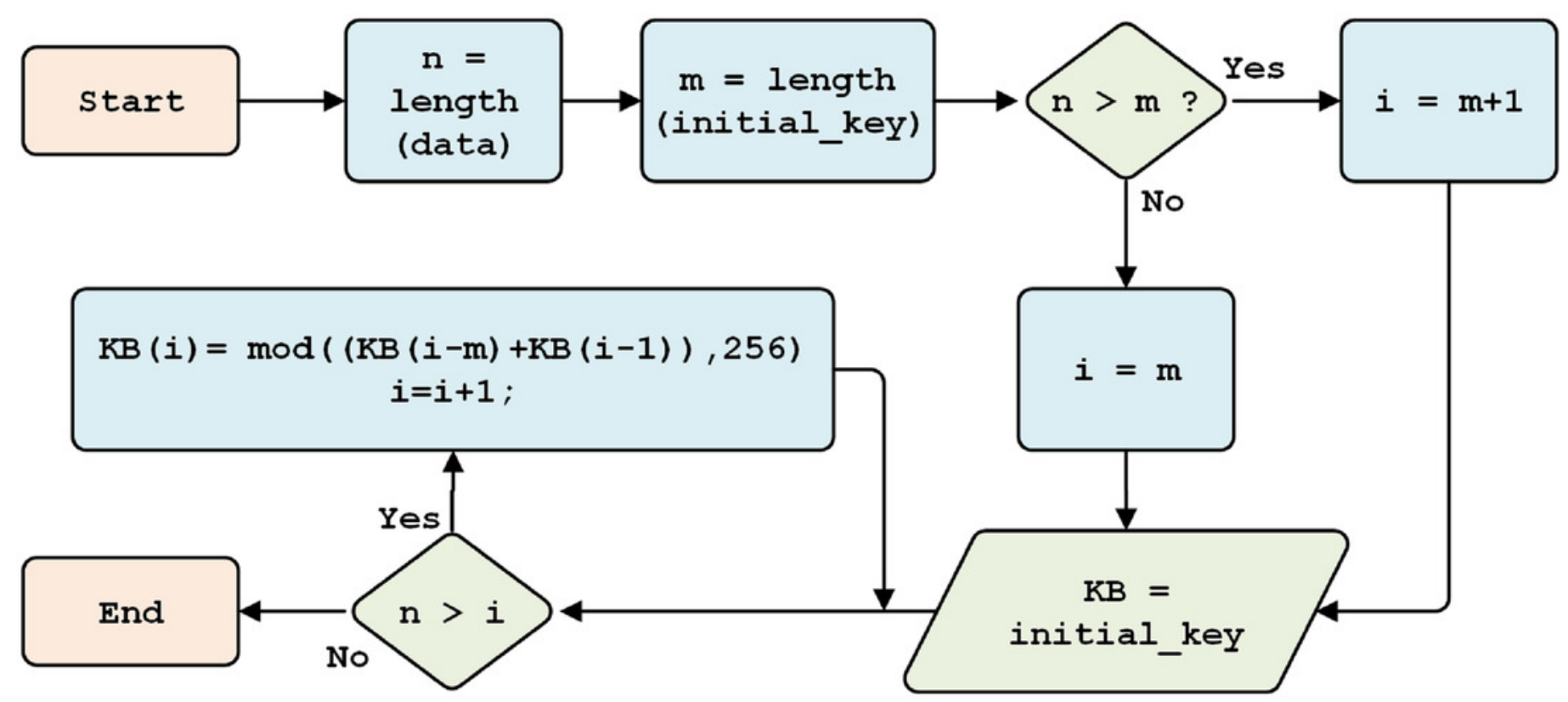


Figure 6

The Beaufort Encryption process

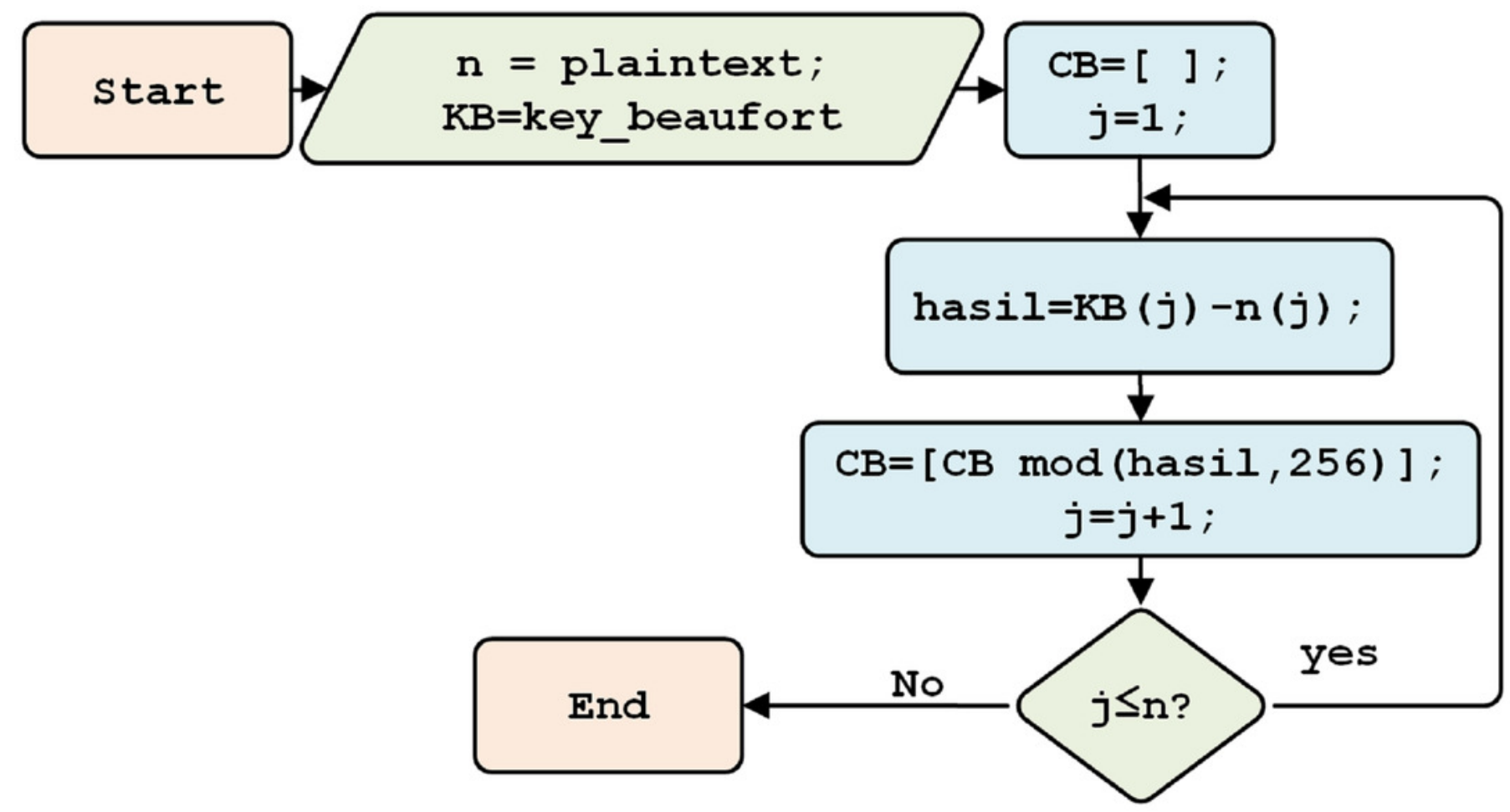


Figure 7

RC4 key Generation 


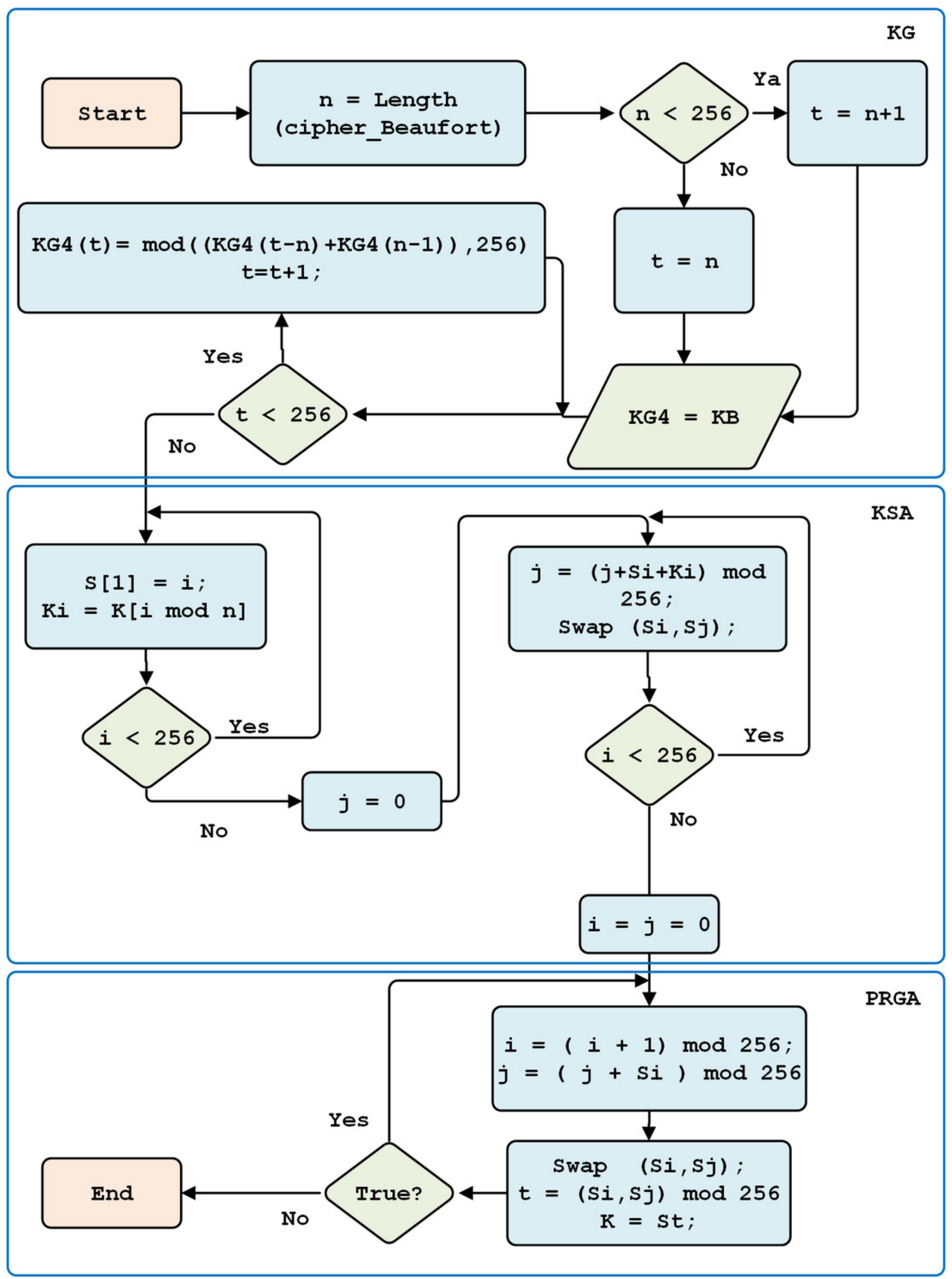


Figure 8

The RC4 Encryption process

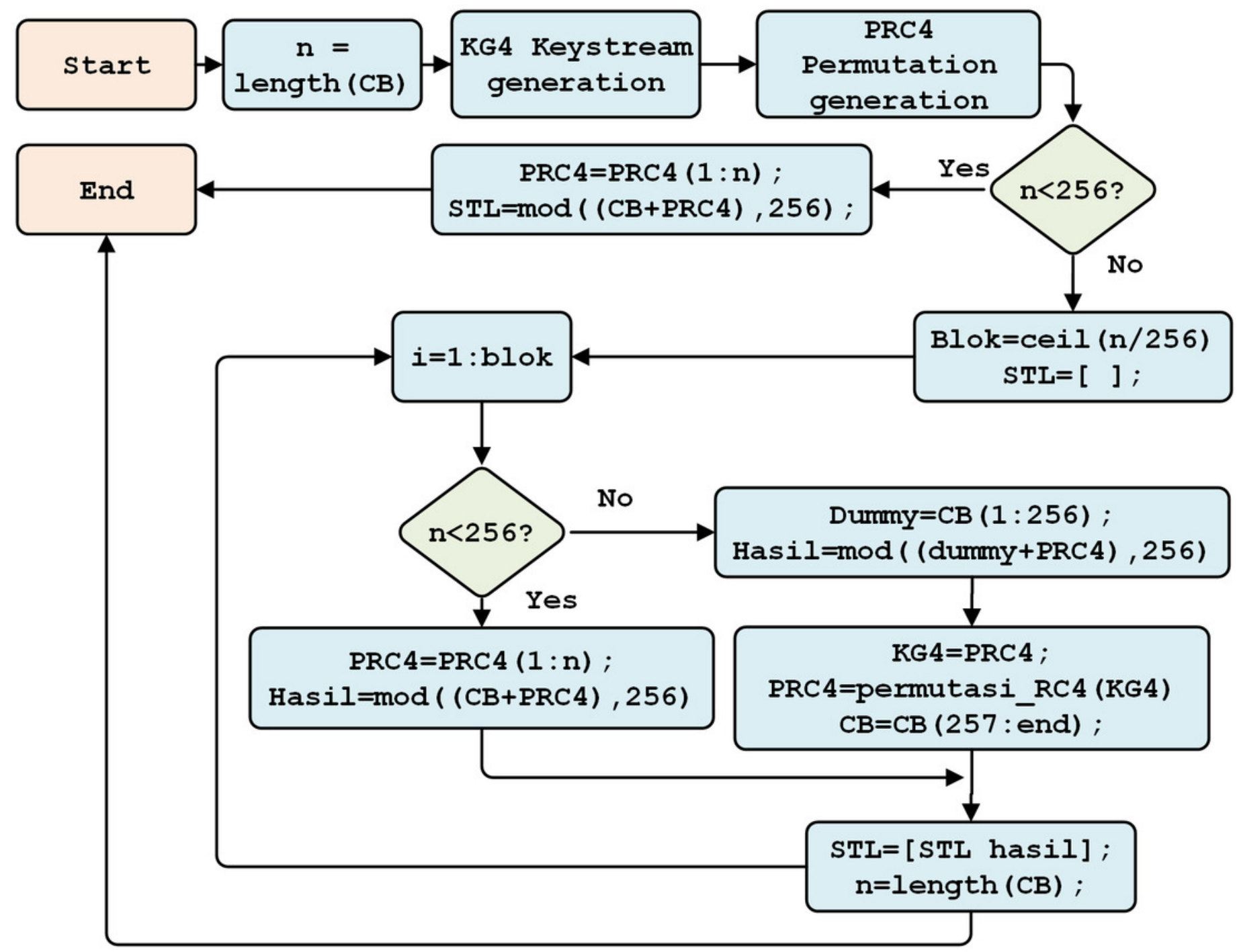


Figure 9

Initial key separation

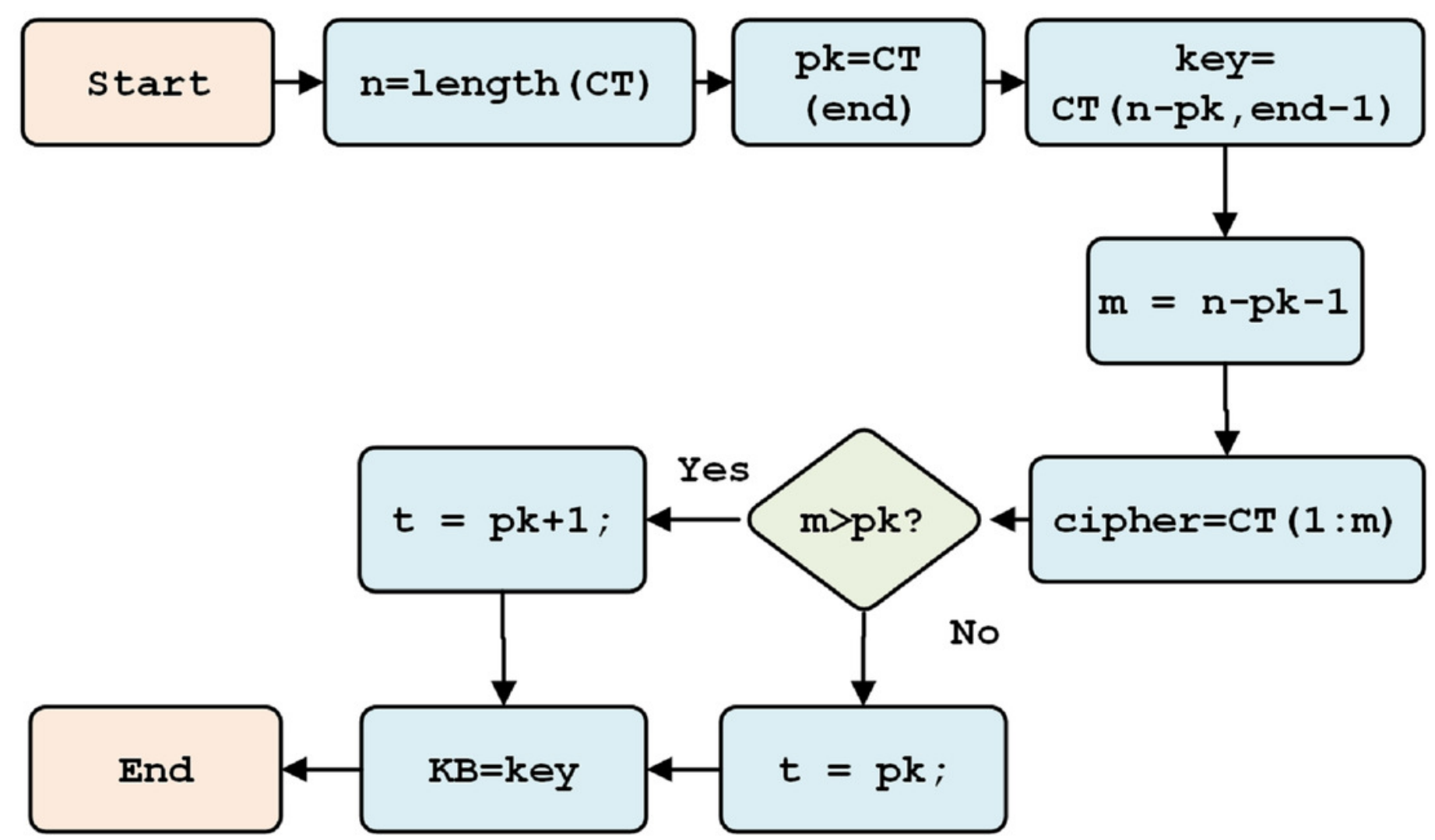


Figure 10

The RC4 decryption process

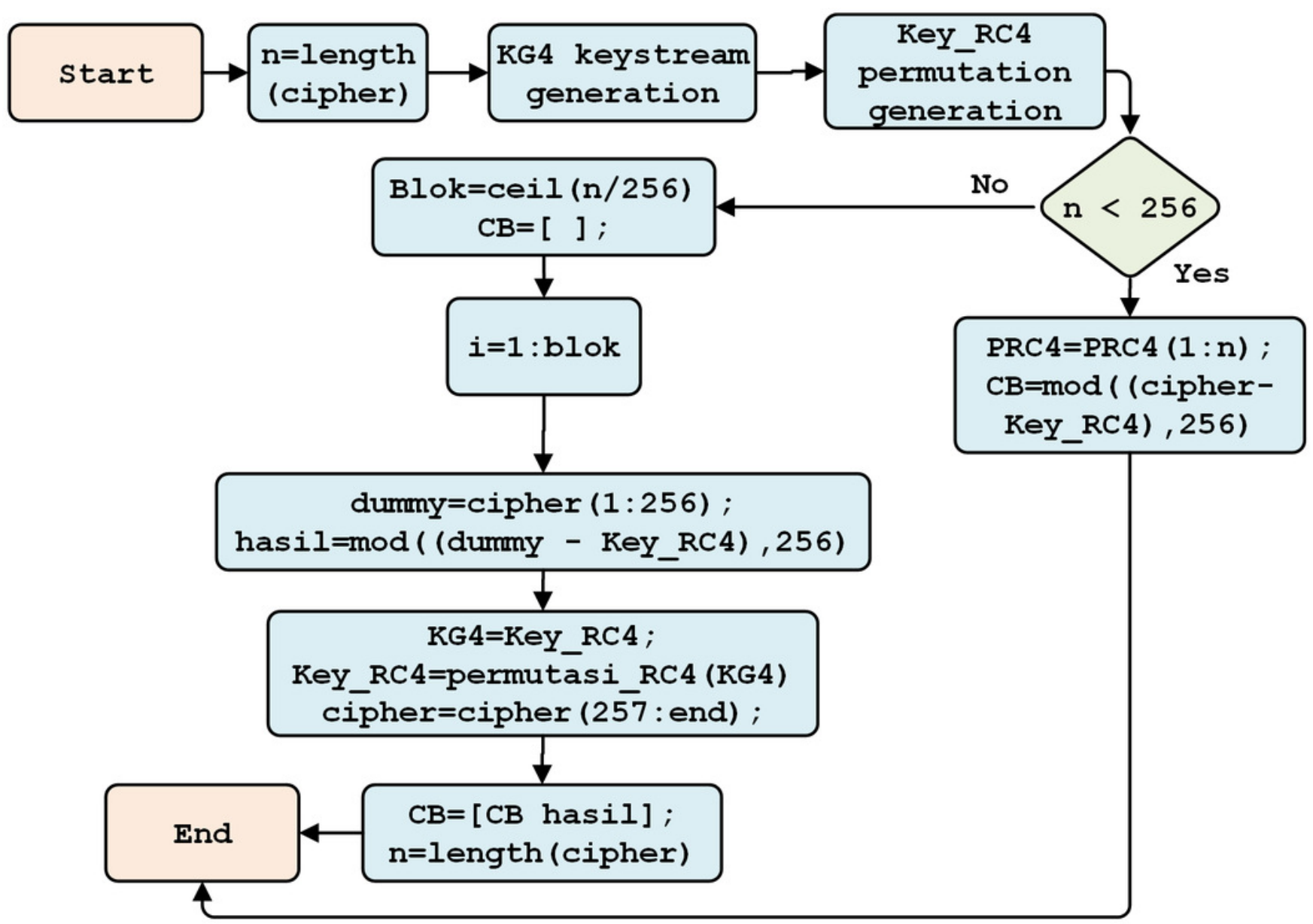


Figure 11

The Beaufort decryption process

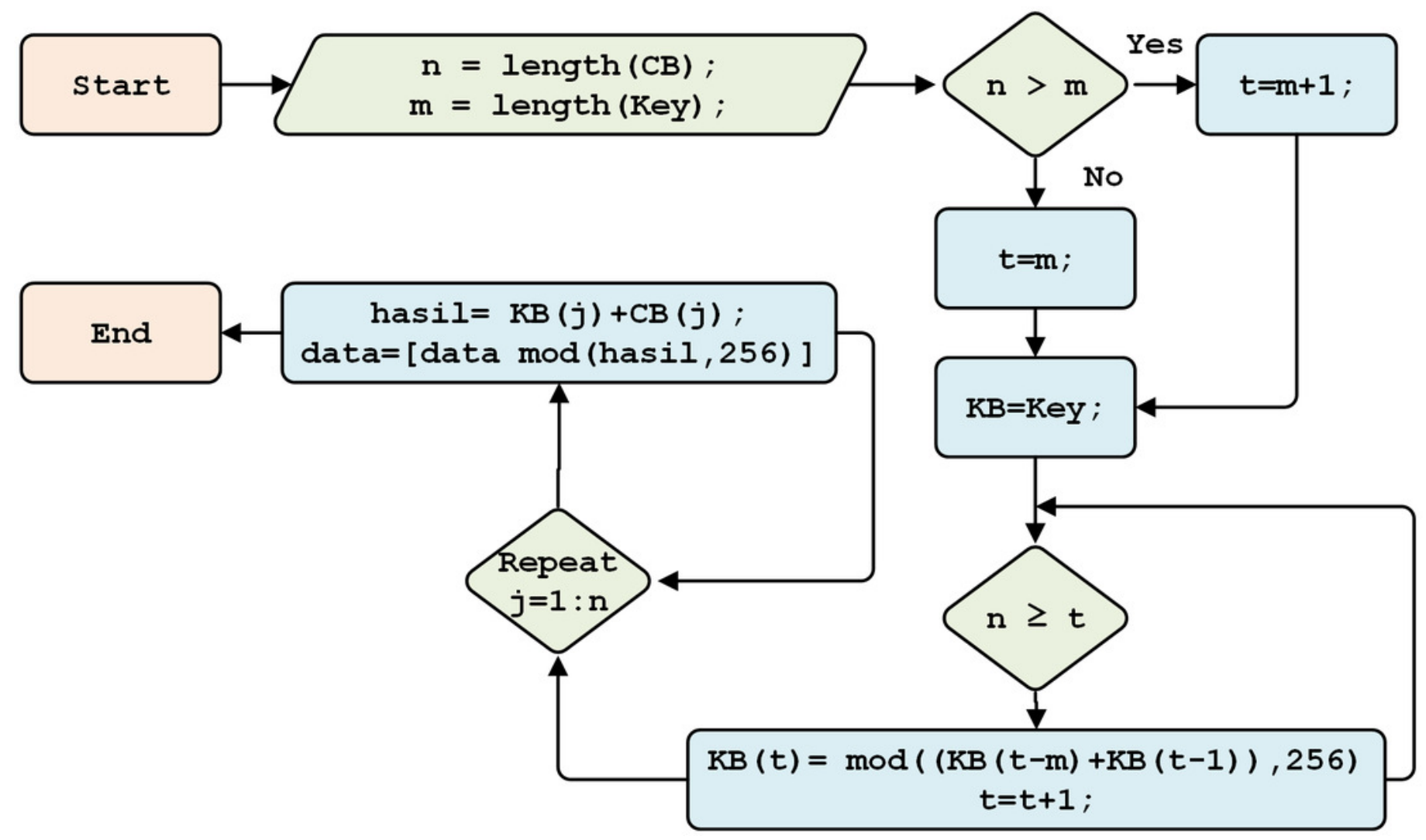


Figure 12

The final model process

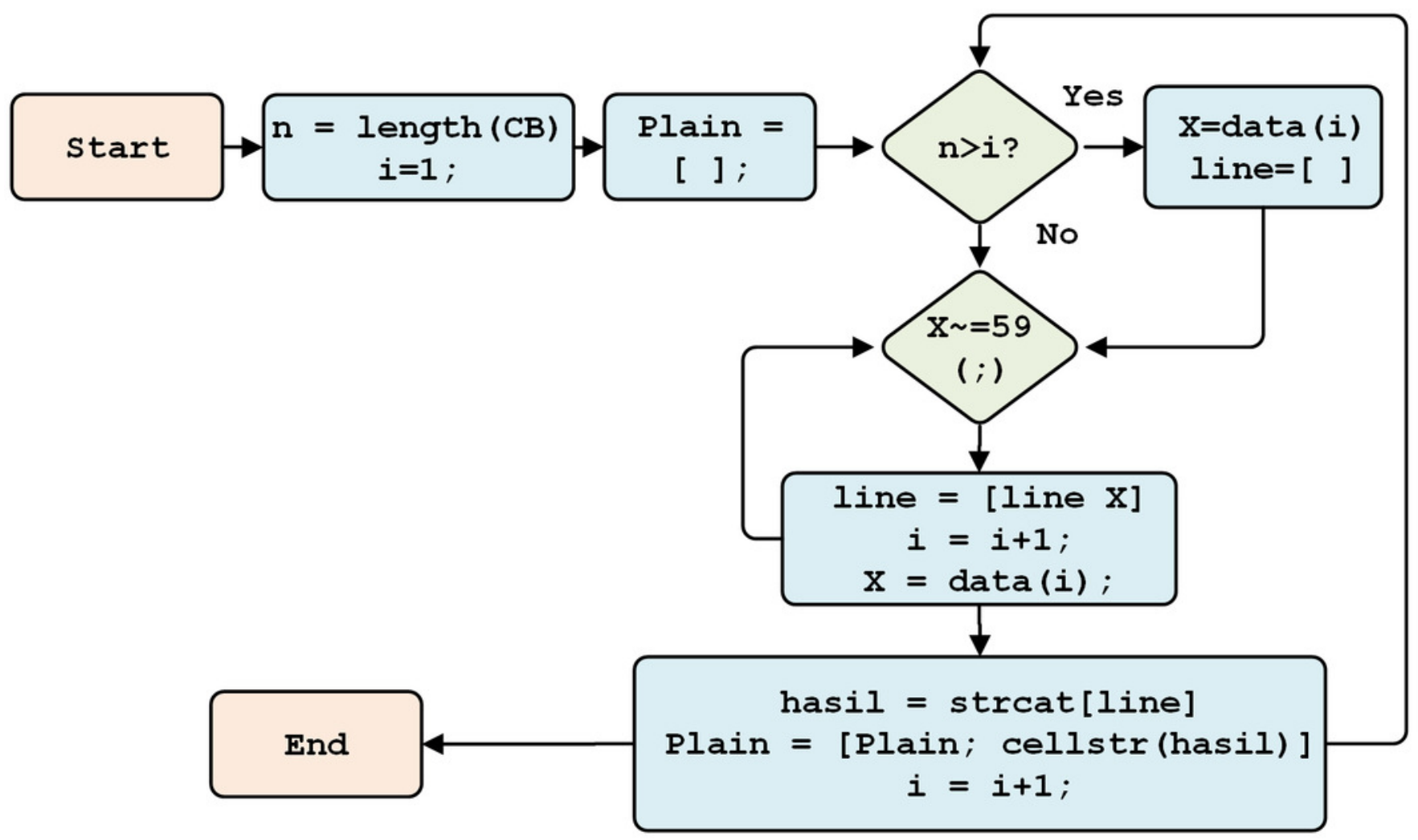


Figure 13

\section{Histogram of plaintext and ciphertext IL data for the first 500 characters}

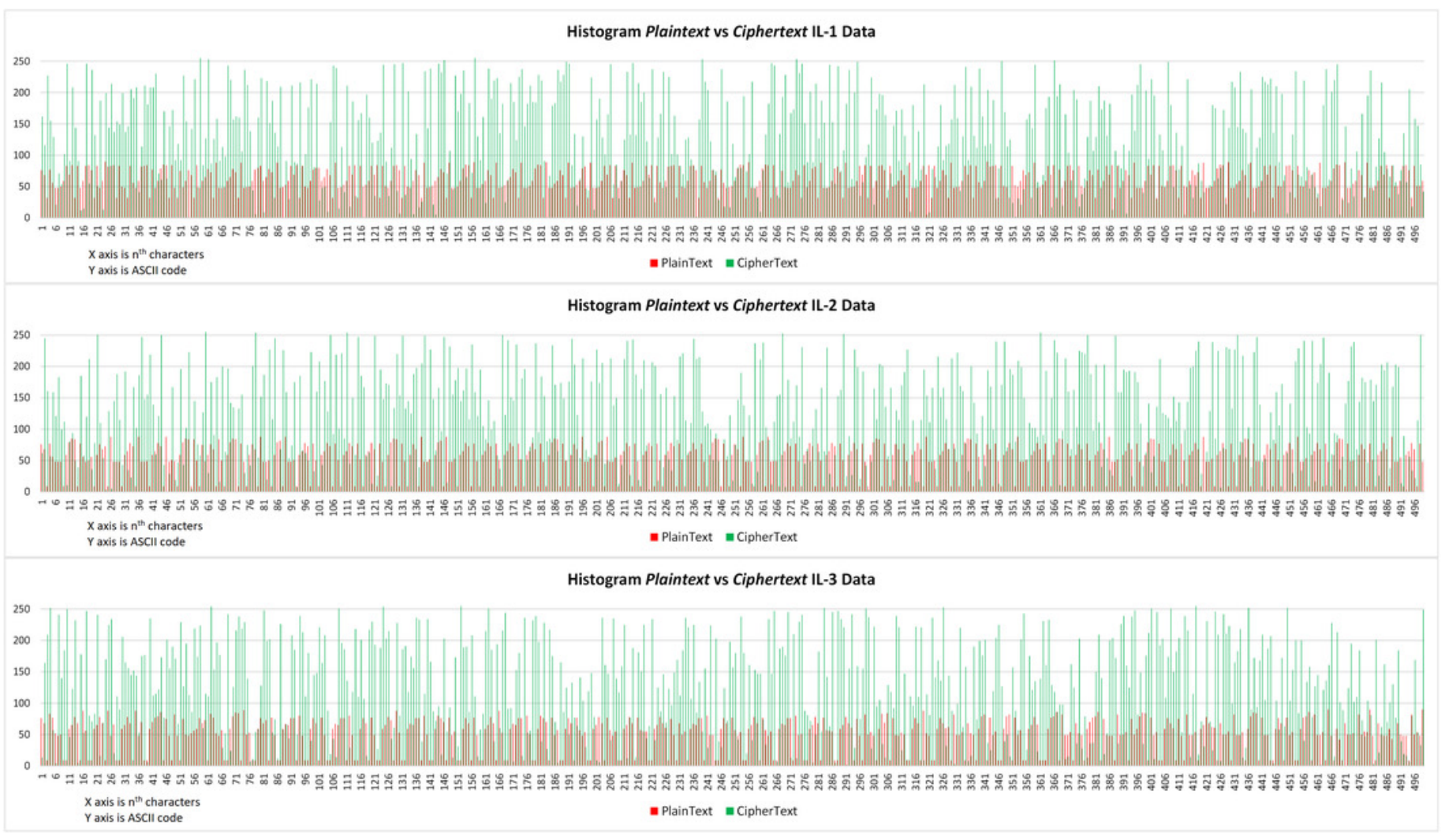




\section{Table 1 (on next page)}

Hybrid security approach to data transmission 
Table 1 Hybrid security approach to data transmission

\begin{tabular}{|c|c|c|c|c|c|}
\hline Study & $\begin{array}{l}\text { Securing Data } \\
\text { Transmission }\end{array}$ & Methods & Performance Measuring & $\begin{array}{l}\text { Provides } \\
\text { layered } \\
\text { security }\end{array}$ & $\begin{array}{l}\text { Provide } \\
\text { security } \\
\text { analysis }\end{array}$ \\
\hline $\begin{array}{l}\text { N. Hong } \\
{[54]}\end{array}$ & $\sqrt{ }$ & $\begin{array}{l}\text { Handshake agreement } \\
\text { (SM2) and ECC. }\end{array}$ & $\begin{array}{l}\text { No performance } \\
\text { evaluation. }\end{array}$ & 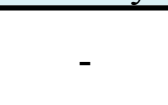 & 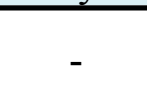 \\
\hline $\begin{array}{l}\text { Altigani } \\
{[56]}\end{array}$ & $\sqrt{ }$ & $\begin{array}{l}\text { AES and } \\
\text { steganography Word } \\
\text { Shift Coding. }\end{array}$ & $\begin{array}{l}\text { Encryption time and } \\
\text { extraction time. }\end{array}$ & $\sqrt{ }$ & - \\
\hline $\operatorname{Xin}[55]$ & $\sqrt{ }$ & $\begin{array}{l}\text { MD5, AES and } \\
\text { ECDH. }\end{array}$ & $\begin{array}{l}\text { Key exchange time, } \\
\text { number of time; key } \\
\text { length, time of signature, } \\
\text { number of signature, } \\
\text { verification time. }\end{array}$ & $\sqrt{ }$ & - \\
\hline $\begin{array}{l}\text { Singh } \\
{[59]}\end{array}$ & $\sqrt{ }$ & $\begin{array}{l}\text { Symmetric } \\
\text { encipherment and } \\
\text { middle value } \\
\text { algorithm. }\end{array}$ & $\begin{array}{l}\text { Encryption and decryption } \\
\text { test }\end{array}$ & $\sqrt{ }$ & - \\
\hline $\begin{array}{l}\text { Purevjav } \\
\quad[52]\end{array}$ & $\sqrt{ }$ & $\begin{array}{l}\text { Symmetric cipher } \\
\text { Ping Pong-128, RSA } \\
\text { and hash function } \\
\text { MD5. }\end{array}$ & $\begin{array}{l}\text { Encryption and decryption } \\
\text { test. }\end{array}$ & $\sqrt{ }$ & - \\
\hline $\begin{array}{l}\text { Z. Hong } \\
{[58]}\end{array}$ & $\sqrt{ }$ & DES and RC4. & No evaluation. & $\sqrt{ }$ & - \\
\hline $\begin{array}{c}\text { Harba } \\
{[53]}\end{array}$ & $\sqrt{ }$ & $\begin{array}{l}\text { AES, RSA and } \\
\text { HMAC. }\end{array}$ & $\begin{array}{l}\text { Ciphertext size, encryption } \\
\text { time }\end{array}$ & $\sqrt{ }$ & - \\
\hline $\begin{array}{l}\text { D'souza } \\
{[57]}\end{array}$ & $\sqrt{ }$ & $\begin{array}{l}\text { AES and Dynamic } \\
\text { Key Generation and } \\
\text { Dynamic S-box } \\
\text { Generation. }\end{array}$ & $\begin{array}{l}\text { Encryption and decryption } \\
\text { test. }\end{array}$ & $\sqrt{ }$ & - \\
\hline $\begin{array}{l}\text { Proposed } \\
\text { Method }\end{array}$ & $\sqrt{ }$ & $\begin{array}{l}\text { Super Encryption } \\
\text { BRC4, Dynamic } \\
\text { Symmetric Four-key- } \\
\text { generation. }\end{array}$ & $\begin{array}{l}\text { Keyspace analysis, } \\
\text { Correlation coefficient } \\
\text { analysis, Information } \\
\text { Entropy analysis, Visual } \\
\text { analysis, Time complexity } \\
\text { analysis, Encryption and } \\
\text { decryption test. }\end{array}$ & $\sqrt{ }$ & $\sqrt{ }$ \\
\hline
\end{tabular}

1 


\section{Table 2 (on next page)}

Keyspace value 
Table 2 Keyspace value (bits)

\begin{tabular}{ccc}
\hline The key length & Keyspace value & Summary \\
\hline 64 bits & $(2)^{64}$ & Not secure \\
128 bits & $(2)^{128}$ & Secure \\
256 bits & $(2)^{256}$ & Secure \\
512 bits & $(2)^{512}$ & Secure \\
1,024 bits & $(2)^{1024}$ & Secure \\
2,048 bits & $(2)^{2048}$ & Secure \\
\hline
\end{tabular}




\section{Table 3 (on next page)}

Pearson correlation coefficient 
Table 3 Pearson correlation coefficient

\begin{tabular}{cccccccccc}
\hline \multicolumn{8}{c}{ Coefficient of correlation (r) } \\
\hline Perfect & High & Moderate & Low & No correlation & Low & Moderate & High & Perfect \\
\hline \multirow{2}{*}{-1} & $\leq-0.90$ & $\leq-0.50$ & $\leq-0.30$ & $\mathbf{- 0 . 2 9} \leq \mathbf{r} \leq+\mathbf{0 . 2 9}$ & $\geq+0.30$ & $\geq+0.50$ & $\geq+0.90$ & 1 \\
\hline
\end{tabular}

1 


\section{Table 4(on next page)}

Correlation coefficient value 
1

Table 4 Correlation coefficient value

\begin{tabular}{lccc}
\hline \multicolumn{1}{c}{ Encoded } & IL1 & IL2 & IL3 \\
\hline Beaufort only & -0.046 & 0.013 & 0.008 \\
RC4 only & -0.093 & -0.126 & 0.437 \\
$\begin{array}{l}\text { Proposed method } \\
\text { (BRC4) }\end{array}$ & -0.010 & 0.006 & 0.001 \\
\hline
\end{tabular}

2 


\section{Table 5 (on next page)}

Information Entropy value 
1

Table 5 Information Entropy value

\begin{tabular}{lccc}
\hline Encoded & IL1 & IL2 & IL3 \\
\hline Beaufort only & 7.77 & 7.91 & 7.94 \\
RC4 only & 7.69 & 7.39 & 5.49 \\
$\begin{array}{l}\text { Proposed method } \\
\text { (BRC4) }\end{array}$ & 7.84 & 7.98 & 7.99 \\
\hline
\end{tabular}

2 


\section{Table 6(on next page)}

Cryptanalysis solutions for the weaknesses of the Vigenere (Beaufort) cipher 
Table 6 Cryptanalysis solutions for the weaknesses of the Vigenere (Beaufort) cipher

\begin{tabular}{|c|c|}
\hline $\begin{array}{c}\text { Weaknesses of the Vigenere (Beaufort) and } \\
\text { Vernam ciphers }\end{array}$ & Proposed method (BRC4) \\
\hline $\begin{array}{l}\text { - The key must be the same length as } \\
\text { plaintext, so the key will be repeated until } \\
\text { it is the same length as the plaintext. }\end{array}$ & $\begin{array}{l}\checkmark \text { The system generates a key using the } \\
\text { keystream generation equation until it has } \\
\text { the same length as the plaintext, and thus } \\
\text { the key is random and not easily solved. }\end{array}$ \\
\hline - $\quad$ The keys have to be random. & $\begin{array}{l}\checkmark \text { The system generates a random initial key } \\
\text { for each session, which is always different. }\end{array}$ \\
\hline - $\quad$ The key must not be reused. & $\begin{array}{l}\checkmark \text { The system generates a random initial key } \\
\text { for each session, which is always different. }\end{array}$ \\
\hline $\begin{array}{l}\text { - The equations used are based on the } \\
\text { standard alphabet (modulo 26). }\end{array}$ & $\begin{array}{l}\checkmark \text { The system uses modulo } 256 \text {, resulting in } \\
\text { increasingly random values of } 256 \text { bytes. }\end{array}$ \\
\hline $\begin{array}{l}\text { - Possible keys are combinations of } \\
\text { lowercase letters, with a maximum length } \\
\text { of } 676 \text { bytes. }\end{array}$ & $\begin{array}{l}\checkmark \text { Possible key variations are derived from } \\
\text { ASCII code, with a maximum length of } \\
65,536 \text { bytes. }\end{array}$ \\
\hline
\end{tabular}




\section{Table 7 (on next page)}

Cryptanalysis solutions for the weaknesses of the RC4 cipher 
Table 7 Cryptanalysis solutions for the weaknesses of the RC4 cipher

\begin{tabular}{|c|c|}
\hline The weaknesses of the RC4 cipher & Proposed method (BRC4) \\
\hline $\begin{array}{l}\text { - The same key tends to be used for all } \\
\text { blocks in the same data package. }\end{array}$ & $\begin{array}{l}\checkmark \text { The system generates a random initial key } \\
\text { (K1), which is different every session, then } \\
\text { generates further keys (K2, K } 3, \mathrm{~K} 4 \text { ) using a } \\
\text { keystream generation equation until it fills up } \\
\text { the array }(\mathrm{K}) \text {. There is thus no repetition of } \\
\text { keys. }\end{array}$ \\
\hline $\begin{array}{l}\text { The original RC4 key is limited to } 40 \text { bits, } \\
\text { and the Initialization Vector (IV) is } \\
\text { limited to } 24 \text { bits. }\end{array}$ & $\begin{array}{l}\checkmark \text { The system generates a random initial key of } \\
\text { up to } 2,048 \text { bits ( } 256 \text { bytes), or even larger. }\end{array}$ \\
\hline $\begin{array}{l}\text { RC4 is effective with large keys, and thus } \\
\text { attacking a PRGA appears ineffective, } \\
\text { even when the most well-known attacks } \\
\text { take over } 2^{700} \text { seconds. It is weak for short } \\
\text { keys, as the key is repeated until it fills the } \\
\text { array }(\mathrm{K}) \text { to a full } 256 \text { bytes. }\end{array}$ & $\begin{array}{l}\text { The system generates a random initial key } \\
\text { (K1), which is different every session, then } \\
\text { generates further keys (K2, K } 3, \mathrm{~K} 4) \text { using a } \\
\text { keystream generation equation until it fills up } \\
\text { the array }(\mathrm{K}) \text {. There is thus no repetition of } \\
\text { keys. }\end{array}$ \\
\hline $\begin{array}{l}\text { - For each PRGA permutation, the value of } \\
\text { the array (S) changes at two locations (at } \\
\text { the most). }\end{array}$ & $\begin{array}{l}\checkmark \text { The system performs different permutations } \\
\text { for every block array, resulting in more varied } \\
\text { random values for array blocks. }\end{array}$ \\
\hline $\begin{array}{l}\text { - Permutation is performed only once for all } \\
\text { blocks formed, forming a pattern that can } \\
\text { be learned by attackers. }\end{array}$ & $\begin{array}{l}\checkmark \text { The system performs different permutations } \\
\text { for every block array to achieve a random } \\
\text { value of } 256 \text { bytes. As such, the system } \\
\text { performs permutations in the first array block, } \\
\text { continues the permutation in the second array } \\
\text { block, third, and so on until the last block, and } \\
\text { as such it generates a random value that varies } \\
\text { for every block array. }\end{array}$ \\
\hline $\begin{array}{l}\text { - It is possible for the same S-Box to be } \\
\text { used. The same pseudorandom value may } \\
\text { be generated repeatedly, as the user key is } \\
\text { repeated to fill the } 256 \text {-byte array. If a key } \\
\text { is used to encrypt } 8 \text { bytes, it will thus be } \\
\text { repeated } 32 \text { times to fill the array. }\end{array}$ & $\begin{array}{l}\checkmark \text { If the key used for permutation is only } 8 \text { bytes } \\
\text { in length, the system uses the keystream } \\
\text { generator to generate fill the key byte array } \\
\text { without repeating the initial key. }\end{array}$ \\
\hline $\begin{array}{l}\text { - An attacker who manages to obtain } \\
\text { multiple ciphertext packets can obtain } \\
\text { several bytes of the original message by } \\
\text { performing XOR operations on two } \\
\text { ciphertext packets. }\end{array}$ & $\begin{array}{l}\text { To perform encryption, the system generates } \\
\text { a random initial key (K1), generates a } \\
\text { keystream (K2), generates a key-scheduling } \\
\text { algorithm (K3), and generates a pseudo- } \\
\text { random key (K4). As such, even if an attacker }\end{array}$ \\
\hline
\end{tabular}




\begin{tabular}{|l|l|}
\hline $\begin{array}{l}\text { For example, if an attacker successfully } \\
\text { intercepts two different encrypted }\end{array}$ & $\begin{array}{l}\text { can obtain the first and the second ciphertext, } \\
\text { XOR operations still cannot be used to } \\
\text { messages that use the same key, the }\end{array}$ \\
$\begin{array}{l}\text { attacker may perform an XOR operation } \\
\text { to remove the key sequence's effect. If the } \\
\text { the initial keys used for the first (K1.1) and } \\
\text { attacker manages to uncover the plaintext } \\
\text { of one encrypted message, the attacker } \\
\begin{array}{l}\text { will easily find other plaintext messages } \\
\text { without knowing the correct key }\end{array}\end{array}$ & $\begin{array}{l}\text { Likewise, K1.1 and K1.2 experience further } \\
\text { generation to produce K4.1 and K4.2, which } \\
\text { sequence. }\end{array}$ \\
\end{tabular}

2 